\title{
An Efficient Route to Tetrahydronaphthols via Addition of ortho-Lithiated Stilbene Oxides to $\alpha, \beta$-Unsaturated Fischer Carbene Complexes
}

\author{
Vito Capriati, ${ }^{a}$ Saverio Florio, ${ }^{a} *$ Renzo Luisi,${ }^{a}$ Filippo Maria Perna,${ }^{a}$ \\ Antonio Salomone, ${ }^{a}$ Francesco Gasparrini ${ }^{b}$
}

${ }^{a}$ Dipartimento Farmaco-Chimico, University of Bari, Via E. Orabona 4, I-70126 - Bari, C.N.R., Istituto di Chimica dei Composti OrganoMetallici "ICCOM", Sezione di Bari, Italy

${ }^{\mathrm{b}}$ Dipartimento di Studi di Chimica e Tecnologia delle Sostanze Biologicamente Attive, Università "La Sapienza”, P.le A. Moro 5, I-00185 Roma, Italy

\section{Experimental part: table of contents}

General

Preparation of Carbene Complexes 4

Spectroscopic data for compound $\mathbf{2 a}$

Spectroscopic data for compounds $\mathbf{4 a - k}$

Preparation of Tetrahydronaphthalenecarboxylates $\mathbf{5}$

Spectroscopic data for compounds 5c,d; 5f,g; 5i

Ortep view of compound $\mathbf{4 g}$

Copy of spectra for compounds $\mathbf{2 a}, \mathbf{4 a - k}$

Copy of spectra for compounds $\mathbf{5 c , d}, \mathbf{5 f}, \mathbf{g}, \mathbf{5 i}-\mathbf{k}$
Pag. S2

Pag. S3

Pag. S3

Pag. S4-S9

Pag. S10

Pag. S10-S13

Pag. S14

Pag. S15-S38

Pag. S39-S52 
General Experimental Methods. Tetrahydrofuran (THF) was freshly distilled under a nitrogen atmosphere over sodium/benzophenone ketyl. Petroleum ether refers to the $40-60{ }^{\circ} \mathrm{C}$ boiling fraction. For the ${ }^{1} \mathrm{H}$ and ${ }^{13} \mathrm{C}$ NMR spectra $\left({ }^{1} \mathrm{H}\right.$ NMR 300, 400, $500 \mathrm{MHz} ;{ }^{13} \mathrm{C}$ NMR 75.4, $\left.100 \mathrm{Mhz}, 125 \mathrm{MHz}\right)$, $\mathrm{CDCl}_{3}$ was used as the solvent. GC-MS spectrometry analyses were performed on a gas-chromatograph HP 5890 (dimethylsilicon capillary column, $30 \mathrm{~m}, 0.25 \mathrm{~mm}$ i.d.) equipped with a mass selective detector operating at $70 \mathrm{eV}(\mathrm{EI})$. MS-ESI analyses were performed on Agilent $1100 \mathrm{LC} / \mathrm{MSD}$ trap system VL. Microanalyses were carried out on an EuroEA 3000 C, H, N, S analyzer. Optical rotations were performed on purified compounds and measured with a Perkin-Elmer 341 polarimeter, using a cell of $1 \mathrm{dm}$ path length at $20^{\circ} \mathrm{C}$; the concentration (c) is expressed in $\mathrm{g} / 100 \mathrm{~mL}$. Melting points (uncorrected) were carried out on an Stuart SMP3 apparatus. Analytical thin layer chromatography (TLC) was carried out on Merck precoated $0.25 \mathrm{~mm}$ thick plates of Kieselgel 60 F254; visualization was accomplished by UV light $(254 \mathrm{~nm})$ or by spraying with a solution of $5 \%(\mathrm{w} / \mathrm{v})$ ammonium molybdate and $0.2 \%(\mathrm{w} / \mathrm{v})$ cerium(III) sulfate in $100 \mathrm{ml} 17.6 \%(\mathrm{w} / \mathrm{v})$ aqueous sulphuric acid and heating to $200{ }^{\circ} \mathrm{C}$ for some time until blue spots appear. All reactions involving air-sensitive reagents were performed under nitrogen in oven-dried glassware using syringe-septum cap technique. Enantiomeric purity assay for compund $(+)-\mathbf{4 g}$ and $(+)-5 g$ was carried out with both racemic and optically active substrates using ${ }^{1} \mathrm{H} \mathrm{NMR}\left(500 \mathrm{MHz}, \mathrm{CCl}_{4}\right.$ with $\mathrm{CDCl}_{3}$ as external standard) in the presence of the Chiral Solvating Agent (CSA) (R)-(-)-2,2,2-trifluoro-1-(9-anthryl)ethanol, ${ }^{1}$ $(\mathrm{CSA} /$ epoxide molar ratio $=2 / 1)$. Tungsten complexes $\mathbf{3} \mathbf{a - g},{ }^{2} \mathbf{3 h}^{3}$ and trans-stilbene oxide derivatives 1a-d ${ }^{4}$ were prepared according to the reported procedure. $(S, S)$-o-bromo trans-stilbene oxide $(S, S)$-1a $($ ee $=99 \%)$ was obtained by preparative HPLC separation of the racemic mixture (Chiralcel OD-H column $256 \times 4.6 \mathrm{~mm} \times \mathrm{ID}$; flow: $1 \mathrm{ml} / \mathrm{min}$, eluent: $n$-hexane/IPA 90-95/5).

\footnotetext{
${ }^{1}$ Parker, D. Chem. Rev. 1991, 91, 1441-1457.

${ }^{2}$ Seco, J. M.; Heinen, H. Chem. Ber. 1987, 120, 537-540.

${ }^{3}$ Wulff, W. D.; Bauta, W. E.; Kaesler, R. W.; Lankford, P. J.; Miller, R. A.; Murray, C. K; Yang, D. C. J. Am. Chem. Soc. 1990, 112, 3642-3659.

${ }^{4}$ Akgun, E.; Glinski, M. B.; Dawan, K. L.; Durst, T. J. Org. Chem. 1981, 46, 2730.
} 
Preparation of Carbene Complexes 4. General Procedure. To a stirred solution of $o$-bromo stilbene oxide $1 \mathrm{a}(1.0 \mathrm{mmol})$ in THF $(6 \mathrm{~mL})$, cooled at $-78^{\circ} \mathrm{C}$ (dry ice/acetone bath), $\mathrm{PhLi}(0.9 \mathrm{mmol}, 0.5 \mathrm{~mL}$ of a 1.8 $\mathrm{M}$ solution in dibutylether), was added and the resulting mixture stirred for $45 \mathrm{~min}$. Then a solution of the tungsten complex $3(0.75 \mathrm{mmol})$ in THF $(2 \mathrm{~mL})$ was added dropwise and the mixture stirred for $1 \mathrm{~h}$ at $-78^{\circ} \mathrm{C}$, allowed to warm to $\mathrm{rt}$ and stirred for further 2-4 hours. After this time it was poured into a rounded bottom flask containing $1 \mathrm{~g}$ of silica gel and the solvent evaporated in vacuo. The crude, adsorbed on silica gel, was column chromatographed (silica gel; petroleum ether/Et $\mathrm{E}_{2} \mathrm{O}$ $9 / 1 \div 95 / 5$ ) to give compounds 4 , which showed the data below.

The intermediate $\mathbf{2 a}$ could be isolated (column chromatography, $\mathrm{R} f, 0.6$, ethyl acetate/petroleum ether, $8 / 2$ ) by quenching the reaction at short reaction time with sat. $\mathrm{NH}_{4} \mathrm{Cl}$.<smiles>COC(=O)CC(c1ccccc1C1O[C@H]1c1ccccc1)c1cccn1C</smiles>

$\left(2^{\prime} R^{*}, 3^{\prime} R^{*}, 3 R^{*}\right)$-Pentacarbonyl-\{3-( $N$-methylpyrrol-2-yl)-3-[2-(3-phenyloxiran-2-yl)phenyl] methoxypropylidene\}tungsten (2a): yellow solid, mp $135-137{ }^{\circ} \mathrm{C}$ (dec.) (hexane), 53\%; ${ }^{1} \mathrm{H}$ NMR $(500 \mathrm{MHz}) \delta 3.08\left(\mathrm{dd}, J^{2}=18.3, J^{3}=3.9 \mathrm{~Hz}, 1 \mathrm{H}\right), 3.15(\mathrm{~s}, 3 \mathrm{H}), 3.70(\mathrm{~d}, J=1.8 \mathrm{~Hz}, 1 \mathrm{H}), 4.08(\mathrm{~d}, J=$ $1.8 \mathrm{~Hz}, 1 \mathrm{H}), 4.11(\mathrm{~s}, 3 \mathrm{H}), 4.30\left(\mathrm{dd}, J^{2}=18.3, J^{3}=10.3 \mathrm{~Hz}, 1 \mathrm{H}\right), 4.83\left(\mathrm{dd}, J^{2}=10.1 \mathrm{~Hz}, J^{3}=3.9 \mathrm{~Hz} 1\right.$ H), 5.98-5.99 (m, $1 \mathrm{H}), 6.02-6.04(\mathrm{~m}, 1 \mathrm{H}), 6.49-6.50$ (m, $1 \mathrm{H}), 6.81-6.83(\mathrm{~m}, 1 \mathrm{H})$, 7.20-7.41 (m, $8 \mathrm{H})$; ${ }^{13} \mathrm{C}$ NMR (125 MHz) $\delta 33.64,35.2,61.0,61.9,70.3,70.5,106.6,107.6,122.3,124.4,125.2,127.1$, $127.4,128.5,128.6,128.7,132.2,133.6,136.7,140.5,197.0(4 \times C=\mathrm{O}), 202.5(C=\mathrm{O}), 330.3(C=\mathrm{W})$; MS (ESI); m/z 668 [M - H] ; FT-IR (KBr, cm $\left.{ }^{-1}\right)$ 2071, 1935, 1447, 1261, 1007, 951, 708. 
<smiles></smiles>

$\left(1 S^{*}, 2 S^{*}, 3 R^{*}, 4 R^{*}\right)$-Pentacarbonyl $\{[4-h y d r o x y-1-(N$-methyl-2-pyrrolyl)-3-phenyl-1,2,3,4tetrahydronaphthalen-2-yl]methoxymethylene\}tungsten (4a): yellow solid, mp $146-147{ }^{\circ} \mathrm{C}$ (dec.) (hexane), $53 \% ;{ }^{1} \mathrm{H}$ NMR $(500 \mathrm{MHz}) \delta 2.43$ (br s, $\left.1 \mathrm{H}\right), 3.22(\mathrm{~s}, 3 \mathrm{H}), 3.97(\mathrm{dd}, J=3.7,2.1 \mathrm{~Hz}, 1 \mathrm{H})$, $4.19(\mathrm{~s}, 3 \mathrm{H}), 4.42(\mathrm{~d}, J=11.9 \mathrm{~Hz}, 1 \mathrm{H}), 4.98$ (d, $J=2.1 \mathrm{~Hz}, 1 \mathrm{H}), 5.51(\mathrm{dd}, J=11.9,3.7 \mathrm{~Hz}, 1 \mathrm{H}), 5.76$ (m, $1 \mathrm{H}), 5.96(\mathrm{~m}, 1 \mathrm{H}), 6.44(\mathrm{~m}, 1 \mathrm{H}), 6.70(\mathrm{~m}, 2 \mathrm{H}), 6.88(\mathrm{~m}, 1 \mathrm{H}), 7.16-7.31(\mathrm{~m}, 5 \mathrm{H}), 7.41(\mathrm{~m}, 1 \mathrm{H})$. ${ }^{13} \mathrm{C}$ NMR $(125 \mathrm{MHz}) \delta 34.7,36.4,49.4,69.5,71.6,71.7,106.6,109.1,122.7,127.1,127.2,128.1$, 128.9, 129.9, 131.5, 135.8, 137.8, 139.2, $196.9(4 \times C=\mathrm{O}), 202.6(C=\mathrm{O}), 338.7(C=\mathrm{W}) ; \mathrm{MS}(\mathrm{ESI}) ; m / z$ $668[\mathrm{M}-\mathrm{H}]^{-}$. FT-IR $\left(\mathrm{KBr}, \mathrm{cm}^{-1}\right)$ 3471, 2069, 1933, 1451, 1267, 1046, 967, 706.<smiles>COC([C@@H]1[C@H](c2ccccc2)c2ccccc2[C@@H](O)[C@H]1c1ccc(OC)cc1)=[N+]([O-])[O-]</smiles>

$\left(1 R^{*}, 2 S^{*}, 3 R^{*}, 4 R^{*}\right)-P e n t a c a r b o n y l\{[4-h y d r o x y-1-(4-m e t h o x y p h e n y l)-3-p h e n y l-1,2,3,4-$

tetrahydronaphthalen-2-yl]methoxymethylene\}tungsten (4b): yellow oil, 80 \%; ${ }^{1} \mathrm{H}$ NMR (500 $\mathrm{MHz}) \delta 3.42$ (br s, exchanges with $\left.\mathrm{D}_{2} \mathrm{O}, 1 \mathrm{H}\right), 3.74(\mathrm{~s}, 3 \mathrm{H}), 3.89$ (dd like t, $\left.J=3.1,2.8 \mathrm{~Hz}, 1 \mathrm{H}\right), 4.12$ (d, $J=11.5 \mathrm{~Hz}, 1 \mathrm{H}), 4.19$ (s, $3 \mathrm{H}), 5.02$ (d, $J=2.8 \mathrm{~Hz}, 1 \mathrm{H}), 5.32$ (dd, $J=10.5,3.1 \mathrm{~Hz}, 1 \mathrm{H}), 6.70-6.72$ $(\mathrm{m}, 2 \mathrm{H}), 6.76-6.81(\mathrm{~m}, 4 \mathrm{H}), 6.96-698(\mathrm{~m}, 2 \mathrm{H}), 7.16-7.30(\mathrm{~m}, 5 \mathrm{H}) .{ }^{13} \mathrm{C} \mathrm{NMR}(125 \mathrm{MHz}) \delta 43.4,49.2$, 55.2, 69.5, 71.8, 74.8, 113.9, 127.05, 127.1, 128.2, 128.6, 128.7, 129.0, 129.6, 130.4, 135.3, 136.7, 138.3, 140.4, 158.3, $196.9(4 \times C=\mathrm{O}), 202.6(C=\mathrm{O}), 338.4(C=\mathrm{W}) . \mathrm{MS}(\mathrm{ESI}) ; m / z 695[\mathrm{M}-\mathrm{H}]^{-}$. FT-IR (film, $\mathrm{cm}^{-1}$ ) 3417, 2068, 1933, 1608, 1511, 1247, 1034, 757, 701 . 
<smiles>COC(=O)[C@@H]1[C@H](c2ccc(Cl)cc2)c2ccccc2[C@@H](O)[C@H]1c1ccccc1</smiles>

$\left(1 R^{*}, 2 S^{*}, 3 R^{*}, 4 R^{*}\right)$-Pentacarbonyl $\{[1-(4-c h l o r o p h e n y l)-4-h y d r o x y-3-p h e n y l-1,2,3,4-$

tetrahydronaphthalen-2-yl]methoxymethylene\}tungsten (4c): yellow solid, mp $142-150{ }^{\circ} \mathrm{C}\left(\mathrm{Et}_{2} \mathrm{O}\right)$, $74 \%$; ${ }^{1} \mathrm{H}$ NMR (500 MHz) $\delta 2.37$ (br. s, $1 \mathrm{H}$ ), 3.91 (dd like t, $J=3.1 \mathrm{~Hz}, 1 \mathrm{H}$ ), 4.16 (d partially overlapping s at $\delta 4.18, J=11.0,1 \mathrm{H}), 4.18(\mathrm{~s}, 3 \mathrm{H}), 5.01(\mathrm{~d}, J=3.1 \mathrm{~Hz}, 1 \mathrm{H}), 5.31(\mathrm{dd}, J=11.0,3.1$ $\mathrm{Hz}, 1 \mathrm{H}), 6.69-6.70(\mathrm{~m}, 3 \mathrm{H}), 7.03-7.05(\mathrm{~m}, 2 \mathrm{H}), 7.17-7.33(\mathrm{~m}, 8 \mathrm{H}) ;{ }^{13} \mathrm{C} \mathrm{NMR}(125 \mathrm{MHz}) \delta 43.7$, $49.2,69.5,71.7,74.5,127.2,127.3,128.2,128.7,128.9,129.5,129.8,130.8,132.6,136.8,138.0$, 139.6, 141.9, $196.8(4 \times C=\mathrm{O}), 202.4(C=\mathrm{O}), 338.9(C=\mathrm{W})$; MS (ESI); $m / z 699[\mathrm{M}-\mathrm{H}]^{-}$; FT-IR $(\mathrm{KBr}$, $\left.\mathrm{cm}^{-1}\right)$ 3405, 3028, 2923, 2069, 1935, 1489, 1014, 759, 701.<smiles>COC(=O)[C@H]1[C@@H](c2ccccc2)[C@H](O)c2ccccc2[C@H]1c1ccc(C)cc1</smiles>

$\left(1 R^{*}, 2 S^{*}, 3 R^{*}, 4 R^{*}\right)$-Pentacarbonyl $\{[(4-h y d r o x y-3-p h e n y l-1-p$-tolyl-1,2,3,4-tetrahydronaphthalen2-yl]methoxymethylene\}tungsten (4d): yellow solid, mp 143-144 ${ }^{\circ} \mathrm{C}$ (hexane), $72 \%$; ${ }^{1} \mathrm{H}$ NMR (500 MHz) $\delta 2.28(\mathrm{~s}, 3 \mathrm{H}), 2.30$ (br s, exchanges with $\left.\mathrm{D}_{2} \mathrm{O}, 1 \mathrm{H}\right), 3.91-3.92($ br s, $1 \mathrm{H}), 4.15(\mathrm{~d}, J=11.0 \mathrm{~Hz}$, $1 \mathrm{H}), 4.20(\mathrm{~s}, 3 \mathrm{H}), 5.02(\mathrm{~d}, J=2.5 \mathrm{~Hz}, 1 \mathrm{H}), 5.34(\mathrm{dd}, J=11.0,3.0 \mathrm{~Hz}, 1 \mathrm{H}), 6.73(\mathrm{~d}, J=6.5 \mathrm{~Hz}, 2 \mathrm{H})$, $6.82(\mathrm{~d}, J=7.5 \mathrm{~Hz}, 1 \mathrm{H}), 6.95$ and $7.04(2 \times \mathrm{d}, \mathrm{AB}$ system, $J=8.0 \mathrm{~Hz}, 4 \mathrm{H}), 7.18-7.30(\mathrm{~m}, 5 \mathrm{H}), 7.46$ $(\mathrm{d}, J=8.0 \mathrm{~Hz}, 1 \mathrm{H}) ;{ }^{13} \mathrm{C} \mathrm{NMR}(125 \mathrm{MHz}) \delta 21.2,44.2,49.5,69.7,72.1,75.0,127.3,127.34,128.4$, $128.8,129.3,129.4,129.6,129.8,129.9,136.5,137.1,138.6,140.4,140.6,197.1(4 \times C=0), 202.9$ $(C=\mathrm{O}), 340.1(C=\mathrm{W})$; MS (ESI); $m / z 679[\mathrm{M}-\mathrm{H}]^{-}$. FT-IR $\left(\mathrm{KBr}, \mathrm{cm}^{-1}\right)$ 3026, 2915, 2069, 1926, 1451, 1263. Anal. Calcd for $\mathrm{C}_{30} \mathrm{H}_{24} \mathrm{O}_{7} \mathrm{~W}: \mathrm{C}, 52.96 ; \mathrm{H}, 3.56$. Found: C, 53.31; H, 3.27. 
<smiles>COC(=O)[C@@H]1[C@H](c2ccccc2)c2ccccc2[C@@H](O)[C@H]1c1ccccc1</smiles>

$\left(1 R^{*}, 2 S^{*}, 3 R^{*}, 4 R^{*}\right)$-Pentacarbonyl $\{[(4-h y d r o x y-3-p h e n y l-1-o-t o l y l \quad 1,2,3,4$-tetrahydronaphthalen2-yl)methoxymethylene\}tungsten (4e): yellow solid, mp 115-120 ${ }^{\circ} \mathrm{C}$ (dec.) (hexane), $52 \%$; ${ }^{1} \mathrm{H}$ NMR (500 MHz) $\delta 2.12$ (br s, $3 \mathrm{H}), 4.04-4.09$ (2 br s, $4 \mathrm{H}), 4.41$ (br d, $J=11.0,1 \mathrm{H}), 5.02$ (br s, $1 \mathrm{H}), 5.53$ (br s, $1 \mathrm{H}), 7.08-7.45(\mathrm{~m}, 13 \mathrm{H}) ;{ }^{13} \mathrm{C}$ NMR (125 MHz) $\delta 19.2,38.8,49.7,69.4,72.0,73.6,125.7,126.5$, 126.6, 127.0, 127.1, 128.1, 128.5, 128.8, 129.0, 129.6, 129.9, 138.1, 140.1, 140.8, 155.0, 155.8, 196.9 $(4 \times C=\mathrm{O}), 202.4(C=\mathrm{O}), 338.6(C=\mathrm{W})$; MS $(\mathrm{ESI}) ; m / z 679[\mathrm{M}-\mathrm{H}]^{-}$; FT-IR $\left(\mathrm{KBr}, \mathrm{cm}^{-1}\right) 3432,2069$, 1916, 1451, 1269, 1206, 1045, 968, 759.<smiles>COC(=O)[C@H]1[C@@H](c2ccccc2)c2ccccc2[C@H](O)[C@@H]1c1ccccc1</smiles>

$\left(1 S^{*}, 2 R^{*}, 3 R^{*}, 4 R^{*}\right)$-Pentacarbonyl $\{[(1-(2-f u r y l)-4-h y d r o x y-3-p h e n y l-1,2,3,4-$

tetrahydronaphthalen-2-yl]methoxymethylene\}tungsten (4f): yellow solid, mp 84-85 ${ }^{\circ} \mathrm{C}$ (hexane), $63 \%$; ${ }^{1} \mathrm{H}$ NMR (300 MHz) $\delta 2.35$ (br. s, exchanges with $\left.\mathrm{D}_{2} \mathrm{O}, 1 \mathrm{H}\right), 3.91(\mathrm{dd}, J=3.7,3.0 \mathrm{~Hz}, 1 \mathrm{H})$, $4.25(\mathrm{~s}, 3 \mathrm{H}), 4.30(\mathrm{~d}, J=10.4 \mathrm{~Hz}, 1 \mathrm{H}), 5.02$ (d, $J=3.0 \mathrm{~Hz}, 1 \mathrm{H}), 5.42$ (dd, $J=10.4,3.74 \mathrm{~Hz}, 1 \mathrm{H})$, 6.01-6.03 (m, $1 \mathrm{H}), 6.26-6.28$ (m, $1 \mathrm{H}), 6.73-6.76$ (m, $2 \mathrm{H})$ 6.92-6.95 (m, $1 \mathrm{H}), 7.20-7.34$ (m, $6 \mathrm{H})$, 7.49-7.51 (m, 1 H); ${ }^{13} \mathrm{C}$ NMR (125 MHz) $\delta 19.3,37.7,49.1,69.6,71.5,108.1,109.8,127.1,127.5$, $128.2,128.5,128.6,128.9,129.8,136.6,136.7,138.1,142.1,196.7(4 \times C=\mathrm{O}), 202.7(C=0), 335.2$ $(C=\mathrm{W}) ; \mathrm{MS}(\mathrm{ESI}) ; \mathrm{m} / z 655$ [M - H] $]^{-}$FT-IR (film, $\left.\mathrm{cm}^{-1}\right): 3424,3031,2965,2065,1920,1250,1165$, 702. 
<smiles>COC(=O)[C@H]1[C@@H](c2ccccc2)[C@H](O)c2ccccc2[C@H]1c1ccccc1</smiles>

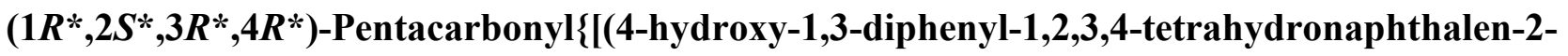

yl]methoxymethylene\}tungsten (4g): yellow solid, mp 132-133 ${ }^{\circ} \mathrm{C}, 71 \%$; ${ }^{1} \mathrm{H}$ NMR (500 MHz) $\delta$ 2.23 (br. s, exchanges with $\left.\mathrm{D}_{2} \mathrm{O}, 1 \mathrm{H}\right), 3.94(\mathrm{dd}, J=3.5,3.0 \mathrm{~Hz}, 1 \mathrm{H}), 4.18(\mathrm{~d}, J=10.6 \mathrm{~Hz}, 1 \mathrm{H}), 4.20$ (s, $3 \mathrm{H}), 5.02$ (d, $J=3.0 \mathrm{~Hz}, 1 \mathrm{H}), 5.37$ (dd, $J=10.6,3.0 \mathrm{~Hz}, 1 \mathrm{H}), 6.72-6.82$ (m, $3 \mathrm{H}), 7.06-7.29$ (m, $10 \mathrm{H}), 7.44-7.50(\mathrm{~m}, 1 \mathrm{H}) ;{ }^{13} \mathrm{C} \mathrm{NMR}(125 \mathrm{MHz}) \delta 44.6,49.5,69.7,72.1,74.8,127.0,127.4,128.4$, $128.8_{0}, 128.8_{4}, 128.8_{6}, 129.3,129.8,129.9_{1}, 129.9_{3}, 137.1,138.6,140.5,143.6,197.1(4 \times C=\mathrm{O}), 202.9$ $(C=\mathrm{O}), 339.4(C=\mathrm{W})$; MS (ESI); $m / z 581\left[\mathrm{M}-(\mathrm{CO})_{3}-\mathrm{H}\right]^{-}$; FT-IR (film, $\left.\mathrm{cm}^{-1}\right): 3415,3030,2974$, 2067, 1917, 1480, 1256, 751. Anal. Calcd for $\mathrm{C}_{29} \mathrm{H}_{22} \mathrm{O}_{7} \mathrm{~W}$ : C, 52.27; H, 3.33. Found: C, 52.59; H, 3.18. (+)-(1S,2R,3S,4S)-4g: $72 \% ;[\alpha]^{20}=+243\left(c 1, \mathrm{CHCl}_{3}\right)$.<smiles>COC(=O)[C@H]1[C@@H](C)c2ccccc2[C@H](O)[C@H]1c1ccccc1</smiles>

$\left(1 S^{*}, 2 S^{*}, 3 R^{*}, 4 R^{*}\right)$-Pentacarbonyl $\{[(4-h y d r o x y-1-m e t h y-3-p h e n y l-1,2,3,4-t e t r a h y d r o n a p h t h a l e n-$

2-yl]methoxymethylene\}tungsten (4h): yellow solid, mp $109-112{ }^{\circ} \mathrm{C}$ (hexane), $71 \%$; ${ }^{1} \mathrm{H}$ NMR (500 MHz) $\delta 1.38(\mathrm{~d}, J=6.7 \mathrm{~Hz}, 3 \mathrm{H}), 2.13$ (br. s, exchanges with $\mathrm{D}_{2} \mathrm{O}, 1 \mathrm{H}$ ), 3.08 (dq, like pentet, $J=7.3$, $6.7 \mathrm{~Hz}, 1 \mathrm{H}), 3.6(\mathrm{dd}$ like t, $J=4.3 \mathrm{~Hz}, 1 \mathrm{H}), 4.30$ (s, $3 \mathrm{H}), 4.84(\mathrm{dd}, J=7.3,4.2 \mathrm{~Hz}, 1 \mathrm{H}), 5.00(\mathrm{~d}, J=$ $4.8 \mathrm{~Hz}, 1 \mathrm{H}), 6.79-6.81(\mathrm{~m}, 2 \mathrm{H}), 7.20-7.34(\mathrm{~m}, 6 \mathrm{H}), 7.44-7.48(\mathrm{~m}, 1 \mathrm{H}) ;{ }^{13} \mathrm{C} \mathrm{NMR}(125 \mathrm{MHz}) \delta$ 20.9, 32.5, 49.2, 69.9, 71.5, 75.3, 126.7, 127.1, 127.3, 128.3, 128.4, 129.0, 129.2, 136.6, 138.8, 140.4, $197.1(4 \times C=\mathrm{O}), 202.9(C=\mathrm{O}), 337.4(C=\mathrm{W})$; MS (ESI); $m / z 603[\mathrm{M}-\mathrm{H}]^{-}$; FT-IR $\left(\mathrm{KBr}, \mathrm{cm}^{-1}\right): 3358$, 2069, 1941, 1444, 1249, 1042, 972, 769, 704. 
<smiles>COC(=O)[C@H]1[C@@H](c2ccc(C)cc2)[C@H](O)c2ccccc2[C@H]1c1ccc(Cl)cc1</smiles>

$\left(1 R^{*}, 2 S^{*}, 3 R^{*}, 4 R^{*}\right)$-Pentacarbonyl $\{[1-(4-c l o r o p h e n y l)-4-h y d r o x y-3-(p-t o l y l) 1,2,3,4-$

tetrahydronaphthalen-2-yl]methoxymethylene\}tungsten (4i) yellow solid, mp 140-141 ${ }^{\circ} \mathrm{C}, 83 \%$; ${ }^{1} \mathrm{H}$ NMR (500 MHz) $\delta 2.36(\mathrm{~s}, 3 \mathrm{H}), 2.39$ (br. s, exchanges with $\left.\mathrm{D}_{2} \mathrm{O}, 1 \mathrm{H}\right) 3.90$ (dd like t, $J=3.1 \mathrm{~Hz}, 1$ H), $4.18(\mathrm{~d}, J=10.4 \mathrm{~Hz}, 1 \mathrm{H}), 4.24(\mathrm{~s}, 3 \mathrm{H}), 5.01(\mathrm{~d}, J=3.0 \mathrm{~Hz}, 1 \mathrm{H}), 5.32(\mathrm{dd}, J=10.4,3.0 \mathrm{~Hz}, 1 \mathrm{H})$, $6.59(\mathrm{~d}, J=7.9 \mathrm{~Hz}, 2 \mathrm{H}) ; 6.75$ (d, $J=7.9 \mathrm{~Hz}, 1 \mathrm{H}), 6.99-7.04$ (m, 4 H), 7.20-7.33 (m, 4 H), 7.47 (d, J $=7.9 \mathrm{~Hz}, 1 \mathrm{H}) ;{ }^{13} \mathrm{C}$ NMR $(125 \mathrm{MHz}) \delta 21.0,43.7,48.9,69.6,71.8,74.5,127.3,128.6_{1}, 128.6_{3}, 128.8$, $128.9,129.8,130.8,132.5,134.8,136.8,136.9,139.6,142.0,196.8(4 \times C=0), 202.4(C=0), 339.1$ $(C=\mathrm{W}) ; \mathrm{MS}(\mathrm{ESI}) ; \mathrm{m} / z 713$ [M - H] $]^{-}$FT-IR (film, $\mathrm{cm}^{-1}$ ): 3429, 3033, 2971, 2066, 1921, 1525, 1247, 798.<smiles>COC(C1C(c2cc(OC)ccc2OC)[C@H](O)c2ccccc2[C@H]1c1ccccc1)=[N+]([O-])OC</smiles>

$\left(1 R^{*}, 2 S^{*}, 3 R^{*}, 4 R^{*}\right)-P e n t a c a r b o n y l\{[4-h y d r o x y-3-(2,5-d i m e t h o x y p h e n y l)-1-p h e n y l-1,2,3,4-$

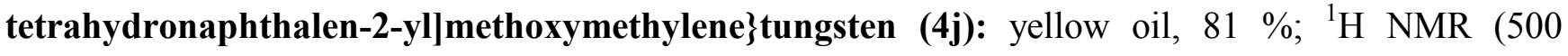
MHz) $\delta 2.37$ (br. s, exchanges with $\left.\mathrm{D}_{2} \mathrm{O}, 1 \mathrm{H}\right), 3.44(\mathrm{~s}, 3 \mathrm{H}), 3.74(\mathrm{~s}, 3 \mathrm{H}), 4.12(\mathrm{~d}, J=11.2 \mathrm{~Hz}, 1 \mathrm{H})$, $4.21(\mathrm{~s}, 3 \mathrm{H}), 4.28(\mathrm{dd}, J=3.1,2.3 \mathrm{~Hz}, 1 \mathrm{H}), 4.87(\mathrm{~d}, J=2.3 \mathrm{~Hz}, 1 \mathrm{H}), 5.40(\mathrm{dd}, J=11.2,3.1 \mathrm{~Hz}, 1 \mathrm{H})$, $6.14(\mathrm{~d}, J=2.9 \mathrm{~Hz}, 1 \mathrm{H})$; 6.69-6.9 (m, $3 \mathrm{H}), 7.04-7.06(\mathrm{~m}, 2 \mathrm{H}), 7.16-7.27$ (m, 5 H), 7.46 (d, $J=7.6$ $\mathrm{Hz}, 1 \mathrm{H}) ;{ }^{13} \mathrm{C} \mathrm{NMR}(125 \mathrm{MHz}) \delta 39.6,44.7,55.0,56.2,69.2,71.9,73.8,110.8,112.1,114.3,126.6$, $127.1,128.1,128.3,128.4,129.5,129.7,137.0,140.6,142.8,151.3,153.1,197.2(4 \times C=0), 203.2$ $(C=\mathrm{O}), 337.6(C=\mathrm{W})$; MS (ESI); $m / z 725$ [M - H] ; FT-IR (film, $\mathrm{cm}^{-1}$ ): 3441, 3031, 2966, 2061, 1927, 1531, 1260 1247, 1119, 798, 700 . 
<smiles>COC([C@H]1[C@H](c2ccccc2)c2ccccc2[C@H](O)[C@H]1c1ccc(C(F)(F)F)cc1)=[N+]([O-])OC</smiles>

$\left(1 R^{*}, 2 S^{*}, 3 R^{*}, 4 R^{*}\right)$-Pentacarbonyl $\{[4-h y d r o x y-1-p h e n y l-3-(4-t r i f l u o r o m e t h y l p h e n y l) 1,2,3,4-$ tetrahydronaphthalen-2-yl]methoxymethylene\}tungsten (4k): Yellow oil, 78 \%. ${ }^{1} \mathrm{H}$ NMR (400 MHz) $\delta 3.98$ (dd like t, $J=3.5,2.6 \mathrm{~Hz}, 1 \mathrm{H}), 4.13(\mathrm{~d}, J=11.3 \mathrm{~Hz}, 1 \mathrm{H}), 4.19$ (s, $3 \mathrm{H}), 4.97$ (d, $J=2.6$ $\mathrm{Hz}, 1 \mathrm{H}), 5.14$ (br. s, exchanges with $\left.\mathrm{D}_{2} \mathrm{O}, 1 \mathrm{H}\right), 5.39$ (dd, $\left.J=11.3,3.5 \mathrm{~Hz}, 1 \mathrm{H}\right), 6.80(\mathrm{~d}, J=7.8 \mathrm{~Hz}, 1$ $\mathrm{H}), 6.84(\mathrm{~d}, J=7.8 \mathrm{~Hz}, 2 \mathrm{H}), 7.05-7.07(\mathrm{~m}, 2 \mathrm{H}), 7.17-7.31(\mathrm{~m}, 7 \mathrm{H}), 7.45(\mathrm{~d}, J=7.8 \mathrm{~Hz}, 1 \mathrm{H}) ;{ }^{13} \mathrm{C}$ $\operatorname{NMR}(125 \mathrm{MHz}) \delta 44.2,48.9,69.4,71.5,74.1,125.0\left(\mathrm{q}, J_{C-F}^{3}=3.9 \mathrm{~Hz}\right), 125.5\left(\mathrm{q}, J_{C-F}^{3}=3.9 \mathrm{~Hz}\right)$, 126.1, 126.8, 127.3, $127.4\left(\mathrm{q}, J_{C-F}^{1}=198.3 \mathrm{~Hz}\right), 128.1,128.6,129.3,129.4,129.5\left(\mathrm{q}, J_{C-F}^{2}=42.9 \mathrm{~Hz}\right)$, 139.1, 139.9, 144.4, $146.0196 .7(4 \times C=\mathrm{O}), 202.4(C=\mathrm{O}), 339.0(C=\mathrm{W}) ; \mathrm{MS}(\mathrm{ESI}) ; m / z 733[\mathrm{M}-\mathrm{H}]^{-}$; FT-IR (film, cm ${ }^{-1}$ ): 3417, 3029, 2966, 2934, 2071, 1930, 1492, 1326, 1265, 1126, 1070, 973, 705. 
Preparation of Tetrahydronaphthalenecarboxylates 5. General Procedure. To a stirred solution of the tungsten complex $5(0.5 \mathrm{mmol})$ in THF $(5 \mathrm{ml})$ at $\mathrm{rt}$ pyridine $N$-oxide was added $(1.5 \mathrm{mmol})$. The resulting mixture was stirred for 24-48 h until the carbene complex (yellow spot on TLC) disappeared. Then the reaction mixture was poured into $20 \mathrm{~mL}$ of sat. aq. $\mathrm{NH}_{4} \mathrm{Cl}$, extracted with $\mathrm{Et}_{2} \mathrm{O}(3 \times 10 \mathrm{~mL})$, dried $\left(\mathrm{Na}_{2} \mathrm{SO}_{4}\right)$, and evaporated under reduced pressure. The crude mixture was column chromatographed (silica gel; petroleum ether/ $\mathrm{Et}_{2} \mathrm{O} 9 / 1 \div 7 / 3$ ) to give tetrahydronaphthalenecarboxylates 5, which showed the following data:<smiles>COC(=O)[C@H]1[C@@H](c2ccccc2)[C@H](O)c2ccccc2[C@H]1c1ccc(Cl)cc1</smiles>

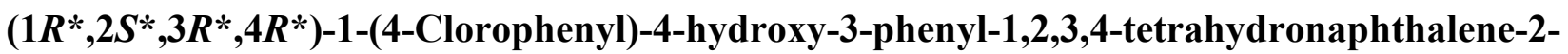
carboxylic acid methyl ester (5c): colorless oil, 81\%; ${ }^{1} \mathrm{H}$ NMR (500 MHz) $\delta 2.28$ (br. s, exchanges with $\left.\mathrm{D}_{2} \mathrm{O}, 1 \mathrm{H}\right), 3.52(\mathrm{~s}, 3 \mathrm{H}), 3.69-3.74(\mathrm{~m}, 2 \mathrm{H}), 4.35(\mathrm{~d}, J=8.9 \mathrm{~Hz}, 1 \mathrm{H}), 5.13(\mathrm{~d}, J=3.3 \mathrm{~Hz}, 1 \mathrm{H})$, $6.84(\mathrm{~d}, \mathrm{~J}=7.8 \mathrm{~Hz}, 1 \mathrm{H}), 6.90(\mathrm{dd}, J=1.6 \mathrm{~Hz}, J=7.7 \mathrm{~Hz}, 2 \mathrm{H}) 7.13-7.29$ (m, $9 \mathrm{H}), 7.45$ (dd, $J=1.0$ $\mathrm{Hz}, J=7.8 \mathrm{~Hz}, 1 \mathrm{H}) ;{ }^{13} \mathrm{C} \mathrm{NMR}(125 \mathrm{MHz}) \delta 43.2,48.6,49.1,51.470 .8,127.2,127.3,128.2,128.4$, 128.5, 128.6, 129.1, 130.2, 131.0, 132.3, 136.8, 138.0, 138.4, 143.2, 173.0; GC-MS (70 eV) m/z (\%) $392\left(\mathrm{M}^{+}, 1\right), 374$ (3), 360 (21), 358 (64), 215 (100), 145 (13), 78 (11); FT-IR (film, cm ${ }^{-1}$ ) 3488, 3041, 2980, 1745, 1513, 1257, 1164, 1089, 811, 706. 
<smiles>COC(=O)[C@H]1[C@@H](c2ccc(C)cc2)[C@@H](O)c2ccccc2[C@H]1c1ccccc1</smiles>

$\left(1 R^{*}, 2 S^{*}, 3 R^{*}, 4 R^{*}\right)-4-H y d r o x y-3-p h e n y l-1-p$-tolyl-1,2,3,4-tetrahydronaphthalene-2-carboxylic acid methyl ester (5d): white solid, mp 65-66 ${ }^{\circ} \mathrm{C}$ (hexane), $55 \% ;{ }^{1} \mathrm{H}$ NMR $(500 \mathrm{MHz}) \delta 2.32(\mathrm{~s}, 3 \mathrm{H})$, 2.39 (br s, excahanges with $\left.\mathrm{D}_{2} \mathrm{O}, 1 \mathrm{H}\right), 3.50(\mathrm{~s}, 3 \mathrm{H}), 3.67-3.75(\mathrm{~m}, 2 \mathrm{H}), 4.35(\mathrm{~d}, J=10.5 \mathrm{~Hz}, 1 \mathrm{H})$, $5.16(\mathrm{~d}, J=4.5 \mathrm{~Hz}, 1 \mathrm{H}), 6.86-6.95(\mathrm{~m}, 3 \mathrm{H}), 7.08(\mathrm{~s}, 4 \mathrm{H}), 7.18-7.29(\mathrm{~m}, 5 \mathrm{H}), 7.46(\mathrm{~d}, J=13.0 \mathrm{~Hz}, 1$ H). ${ }^{13} \mathrm{C}$ NMR (125 MHz) $\delta$ 20.9, 43.7, 48.5, 49.6, 51.3, 70.8, 126.9, 127.2, 128.3, 128.38, 128.4, 128.9, 129.0, 129.4, 130.2, 136.0, 137.1, 138.6, 138.9, 141.6, 173.3. GC-MS (70 eV) m/z (\%) $372\left(\mathrm{M}^{+}\right.$, 5), 354 (60), 295 (68), 263 (100), 221 (50), 196 (98), 165 (28), 91 (23). FT-IR (film, cm ${ }^{-1}$ ) 3406, 3027, 2924, 1741, 1453, 1167, 1036, 806, 701. Anal. Calcd for $\mathrm{C}_{25} \mathrm{H}_{24} \mathrm{O}_{3}$ : C, 80.62; H, 6.49. Found: C, 80.88; H, 6.48.<smiles>COC(=O)[C@@H]1[C@H](c2ccco2)c2ccccc2[C@@H](O)[C@@H]1c1ccccc1</smiles>

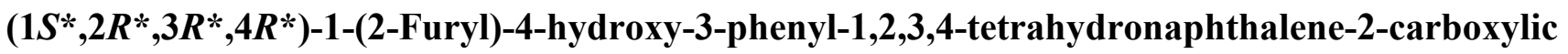
acid methyl ester (5f): colorless oil, $59 \% ;{ }^{1} \mathrm{H}$ NMR $(500 \mathrm{MHz}) \delta 2.13$, (br. s, exchanges with $\mathrm{D}_{2} \mathrm{O}, 1$ H) $3.55(\mathrm{~s}, 3 \mathrm{H}), 3.64(\mathrm{dd}$ like t, $J=4.8 \mathrm{~Hz}, 1 \mathrm{H}), 3.82(\mathrm{dd}, J=7.9,4.4 \mathrm{~Hz}, 1 \mathrm{H}), 4.48(\mathrm{~d}, J=4.4 \mathrm{~Hz}, 1$ H), $5.22(\mathrm{~d}, J=4.9 \mathrm{~Hz}, 1 \mathrm{H}), 6.07(\mathrm{~d}, J=3.1 \mathrm{~Hz}, 1 \mathrm{H}), 6.29(\mathrm{dd}, J=3.1,1.8 \mathrm{~Hz}, 1 \mathrm{H}), 6.98-7.00$ (m, 2 $\mathrm{H}), 7.07(\mathrm{~d}, J=7.3 \mathrm{~Hz}, 1 \mathrm{H}), 7.22-7.33(\mathrm{~m}, 6 \mathrm{H}), 7.52(\mathrm{~d}, J=7.3 \mathrm{~Hz}, 1 \mathrm{H}) ;{ }^{13} \mathrm{C} \mathrm{NMR}(125 \mathrm{MHz})$ $\delta 38.6,46.7,48.3,51.5,70.2,108.3,110.2,126.1,126.2,127.3,127.5,128.3,128.5,129.0,129.2$, 135.3, 137.2, 138.5, 141.8, 173.0; GC-MS (70 eV) m/z (\%) $348\left(\mathrm{M}^{+}, 6\right), 330$ (100), 281 (59), 202 (17), 91 (20), 77 (12); FT-IR (film, cm ${ }^{-1}$ ) 3492, 3044, 3031, 2981, 1737, 1262, 1109, 976, 751, 701. 
<smiles>COC(=O)[C@H]1[C@@H](c2ccccc2)[C@H](O)c2ccccc2[C@H]1c1ccccc1</smiles>

$\left(1 R^{*}, 2 S^{*}, 3 R^{*}, 4 R^{*}\right)-4-H y d r o x y-1,3-d i p h e n y l-1,2,3,4-t e t r a h y d r o n a p h t h a l e n e-2-c a r b o x y l i c \quad$ acid methyl ester (5g): white solid, mp 96-97 ${ }^{\circ} \mathrm{C}\left(\mathrm{Et}_{2} \mathrm{O}\right), 76 \%$ \% ${ }^{1} \mathrm{H}$ NMR $(300 \mathrm{MHz}) \delta 2.16$ (br. s, exchanges with $\left.\mathrm{D}_{2} \mathrm{O}, 1 \mathrm{H}\right), 3.50(\mathrm{~s}, 3 \mathrm{H}), 3.69(\mathrm{dd}, J=8.5,4.4 \mathrm{~Hz}, 1 \mathrm{H}), 3.74$ (dd like t, $J=4.4 \mathrm{~Hz}, 1$ $\mathrm{H}), 4.38(\mathrm{~d}, J=8.5 \mathrm{~Hz}, 1 \mathrm{H}), 5.17(\mathrm{~d}, J=4.4 \mathrm{~Hz}, 1 \mathrm{H}), 6.85-6.98(\mathrm{~m}, 4 \mathrm{H}), 7.06-7.49(\mathrm{~m}, 10 \mathrm{H}) ;{ }^{13} \mathrm{C}$ NMR $(125 \mathrm{MHz}) \delta 44.0,48.5,49.6,51.4,70.8,126.6,127.2,127.3,128.4_{0}, 128.4_{1}, 128.5,128.6$, 129.0, 129.6, 130.2, 137.1, 138.4, 138.8, 144.7, 173.2; GC-MS (70 eV) m/z (\%) $358\left(\mathrm{M}^{+}, 5\right), 340(80)$, 281 (73), 249 (91), 196 (100), 178 (46), 91 (31); FT-IR (film, $\mathrm{cm}^{-1}$ ): 3415, 3023, 2923, 1736, 1492, 1167, 1033, 761, 700. Anal. Calcd for $\mathrm{C}_{24} \mathrm{H}_{22} \mathrm{O}_{3}$ : C, 80.42; H, 6.19. Found: C, 80.73; $\mathrm{H}, 6.22$. $(+)-(1 S, 2 R, 3 S, 4 S)-5 g: 75 \% ;[\alpha]^{20}=+104\left(c 1, \mathrm{CHCl}_{3}\right)$.<smiles>COC(=O)[C@H]1[C@@H](c2ccc(C)cc2)[C@@H](O)c2ccccc2[C@H]1c1ccc(Cl)cc1</smiles>

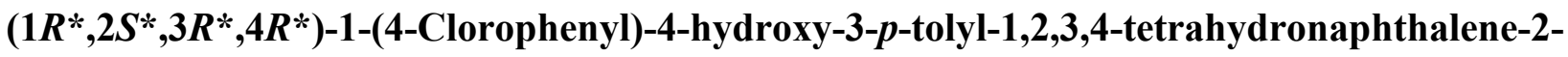

carboxylic acid methyl ester (5i): colorless oil, $79 \% ;{ }^{1} \mathrm{H}$ NMR (500 MHz) $\delta 1.83$ (br. s, exchanges with $\left.\mathrm{D}_{2} \mathrm{O}, 1 \mathrm{H}\right) 2.27(\mathrm{~s}, 3 \mathrm{H}), 3.53(\mathrm{~s}, 3 \mathrm{H}), 3.66-3.71(\mathrm{~m}, 2 \mathrm{H}), 4.34(\mathrm{~d}, J=9.5 \mathrm{~Hz}, 1 \mathrm{H}), 5.10(\mathrm{~d}, J=$ $3.5 \mathrm{~Hz}, 1 \mathrm{H}), 6.77(\mathrm{~d}, J=8.0 \mathrm{~Hz}, 2 \mathrm{H}), 6.84(\mathrm{~d}, J=7.7 \mathrm{~Hz}, 1 \mathrm{H}), 7.00(\mathrm{~d}, J=7.9 \mathrm{~Hz}, 2 \mathrm{H}), 7.14(\mathrm{~d}, J=$ $8.3 \mathrm{~Hz}, 2 \mathrm{H}), 7.22-7.31(\mathrm{~m}, 4 \mathrm{H}), 7.44(\mathrm{~d}, J=7.5 \mathrm{~Hz}, 1 \mathrm{H}) ;{ }^{13} \mathrm{C}$ NMR $(125 \mathrm{MHz}) \delta 20.9,29.6,43.2$, 48.3, 49.1, 71.0, 125.6, 127.2, 128.1, 128.5, 128.6, 129.1, 130.1, 131.0, 132.3, 134.9, 136.9, 137.3, 138.5, 143.3, 173.1; GC-MS (70 eV) m/z (\%) $406\left(\mathrm{M}^{+}, 2\right), 390$ (20), 388 (61), 331 (34), 329 (100), 294 (44), 278 (29), 202 (31), 138 (12); FT-IR (film, cm ${ }^{-1}$ ) 3446, 3033, 2977, 1744, 1521, 1266, 1118, 1011, 809, 701 . 


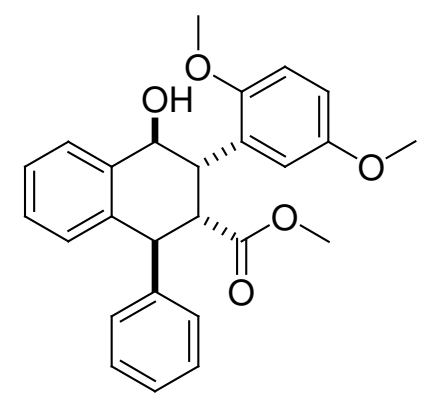

$\left(1 R^{*}, 2 S^{*}, 3 R^{*}, 4 R^{*}\right)-3-(2,5-D i m e t h o x y p h e n y l)-4-h y d r o x y-1-p h e n y l-1,2,3,4-$

tetrahydronaphthalene-2-carboxylic acid methyl ester (5j): colorless oil, 69 \%; ${ }^{1} \mathrm{H}$ NMR (400 MHz) $\delta 3.43(\mathrm{~s}, 3 \mathrm{H}), 3.53(\mathrm{~s}, 3 \mathrm{H}), 3.55(\mathrm{~s}, 3 \mathrm{H}), 3.62(\mathrm{dd}, J=8.5,4.2 \mathrm{~Hz}, 1 \mathrm{H}), 4.05$ (dd like t, $J=$ 4.8, 4.2 Hz, $1 \mathrm{H}), 4.20$ (br. s, exchanges with $\left.\mathrm{D}_{2} \mathrm{O}, 1 \mathrm{H}\right) ; 4.33(\mathrm{~d}, J=8.5 \mathrm{~Hz}, 1 \mathrm{H}), 5.13(\mathrm{~d}, J=4.8 \mathrm{~Hz}$, $1 \mathrm{H}), 6.48(\mathrm{~d}, J=2.4 \mathrm{~Hz}, 1 \mathrm{H}), 6.67-6.70(\mathrm{~m}, 2 \mathrm{H}), 6.84(\mathrm{~d}, J=7.9 \mathrm{~Hz}, 1 \mathrm{H}), 7.13-7.29(\mathrm{~m}, 7 \mathrm{H}), 7.51$ $(\mathrm{d}, J=7.0 \mathrm{~Hz}, 1 \mathrm{H}) ;{ }^{13} \mathrm{C} \mathrm{NMR}(125 \mathrm{MHz}) \delta 39.6,44.8,48.8,52.3,55.0,56.2,71.9,110.7,112.0$, $114.3,126.6,127.1,128.1,128.4,1285,129.6,129.8,137.0,140.6,142.7,151.3,153.0,173.0$; GCMS (70 eV) m/z (\%) $418\left(\mathrm{M}^{+}, 22\right), 400$ (10), 368 (12), 221 (25), 178 (26), 138 (100); FT-IR (film, cm 1) $3515,3039,2976,1744,1266,1262,1117,812,751,701$.<smiles>COC(=O)[C@H]1C(c2ccccc2)c2ccccc2C(O)[C@H]1c1ccc(C(F)(F)F)cc1</smiles>

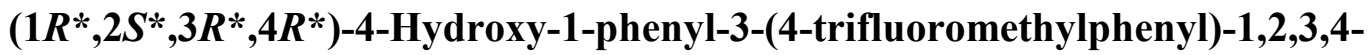

tetrahydronaphthalene-2-carboxylic acid methyl ester (5k): colorless oil, 72 \%; ${ }^{1} \mathrm{H}$ NMR (400 MHz) $\delta 3.19$ (br.s, exchanges with $\left.\mathrm{D}_{2} \mathrm{O}, 1 \mathrm{H}\right) 3.48(\mathrm{~s}, 3 \mathrm{H}), 3.68(\mathrm{~d}, J=10.1 \mathrm{~Hz}, 1 \mathrm{H}) ; 5.14-5.17$ (m, 2 H), 5.42 (br. s, $1 \mathrm{H}), 7.05-7.30(\mathrm{~m}, 10 \mathrm{H}), 7.57(\mathrm{~d}, J=7.8 \mathrm{~Hz}, 1 \mathrm{H}), 7.68(\mathrm{~d}, J=7.8 \mathrm{~Hz}, 2 \mathrm{H}) ;{ }^{13} \mathrm{C}$ $\operatorname{NMR}(125 \mathrm{MHz}) \delta 43.9,49.0,51.3,59.7,72.8,125.5\left(\mathrm{~m}, J_{C-F}^{3}=3.1 \mathrm{~Hz}, 2 C_{\text {ortho }}\right), 126.6,126.8,126.9$ $\left(\mathrm{q}, J_{C-F}^{l}=198.9 \mathrm{~Hz}\right), 128.1,128.3,129.6,129.8\left(\mathrm{q}, J_{C-F}^{2}=34.9 \mathrm{~Hz}\right), 130.1,137.0,139.1,142.8$, 146.1; FT-IR (film, cm ${ }^{-1}$ ) 3501, 3035, 2977, 1737, 1496, 1264, 1161, 1050, 753, 706. 


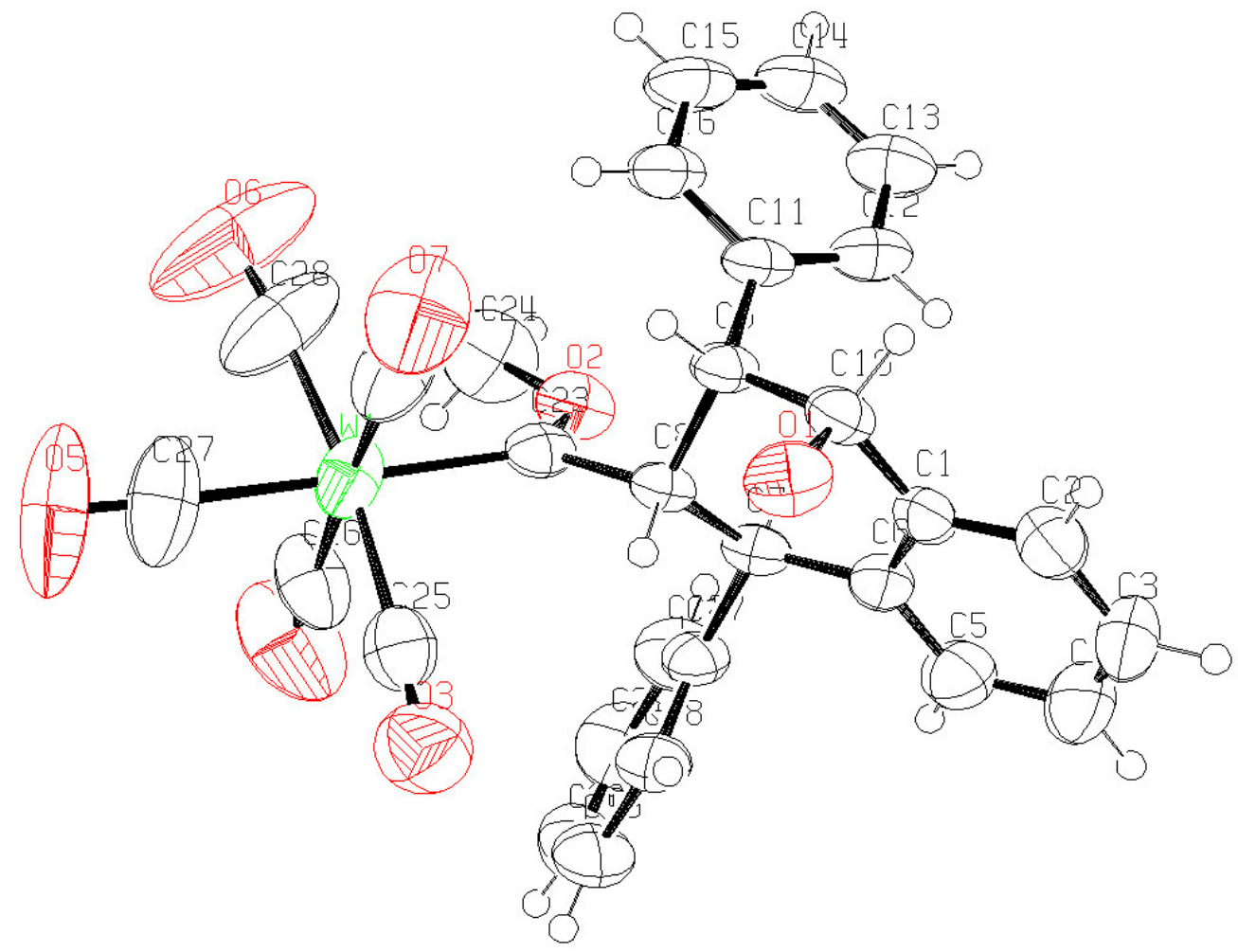

Ortep view of compound $\mathbf{4 g}$ 


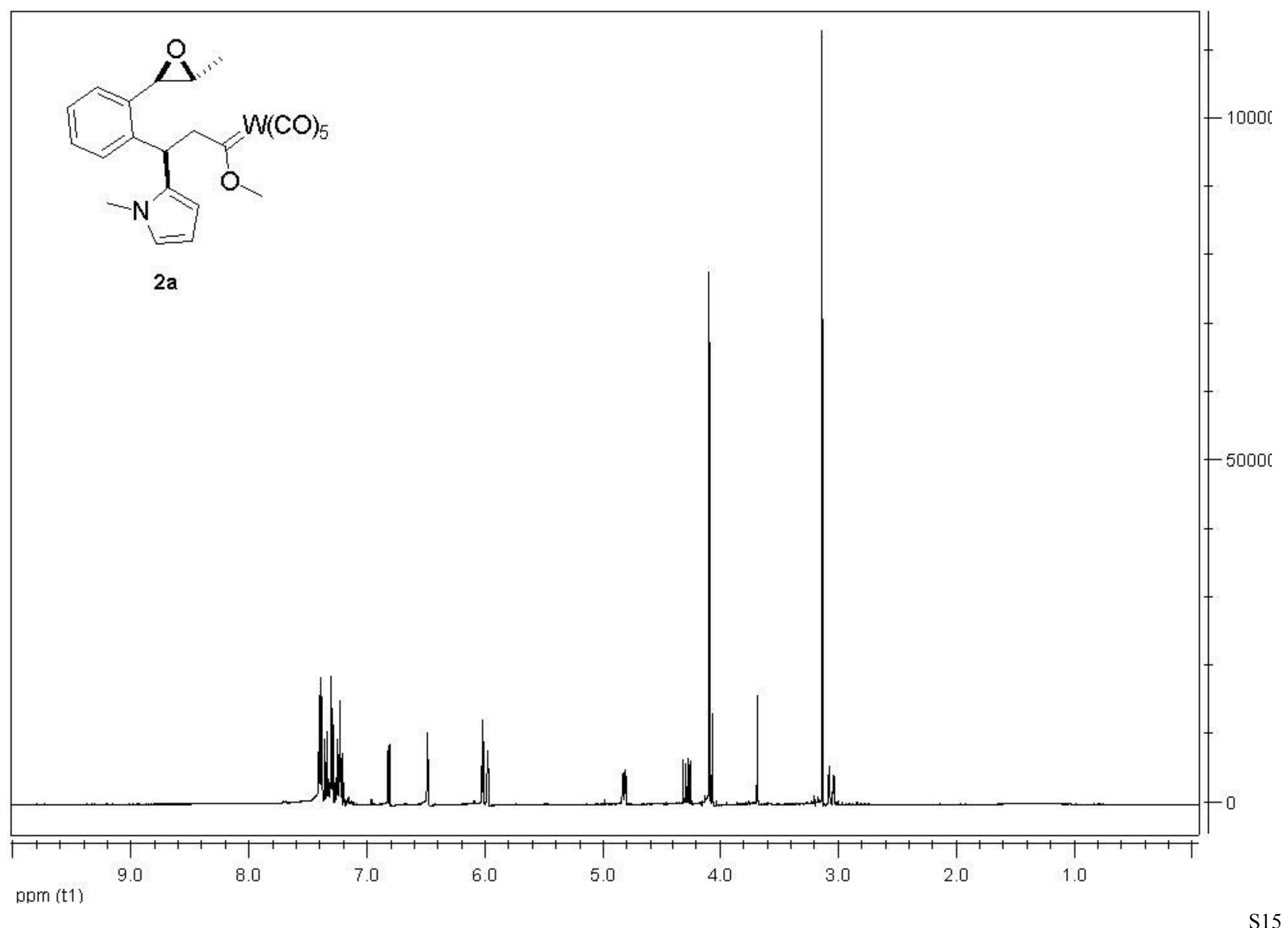




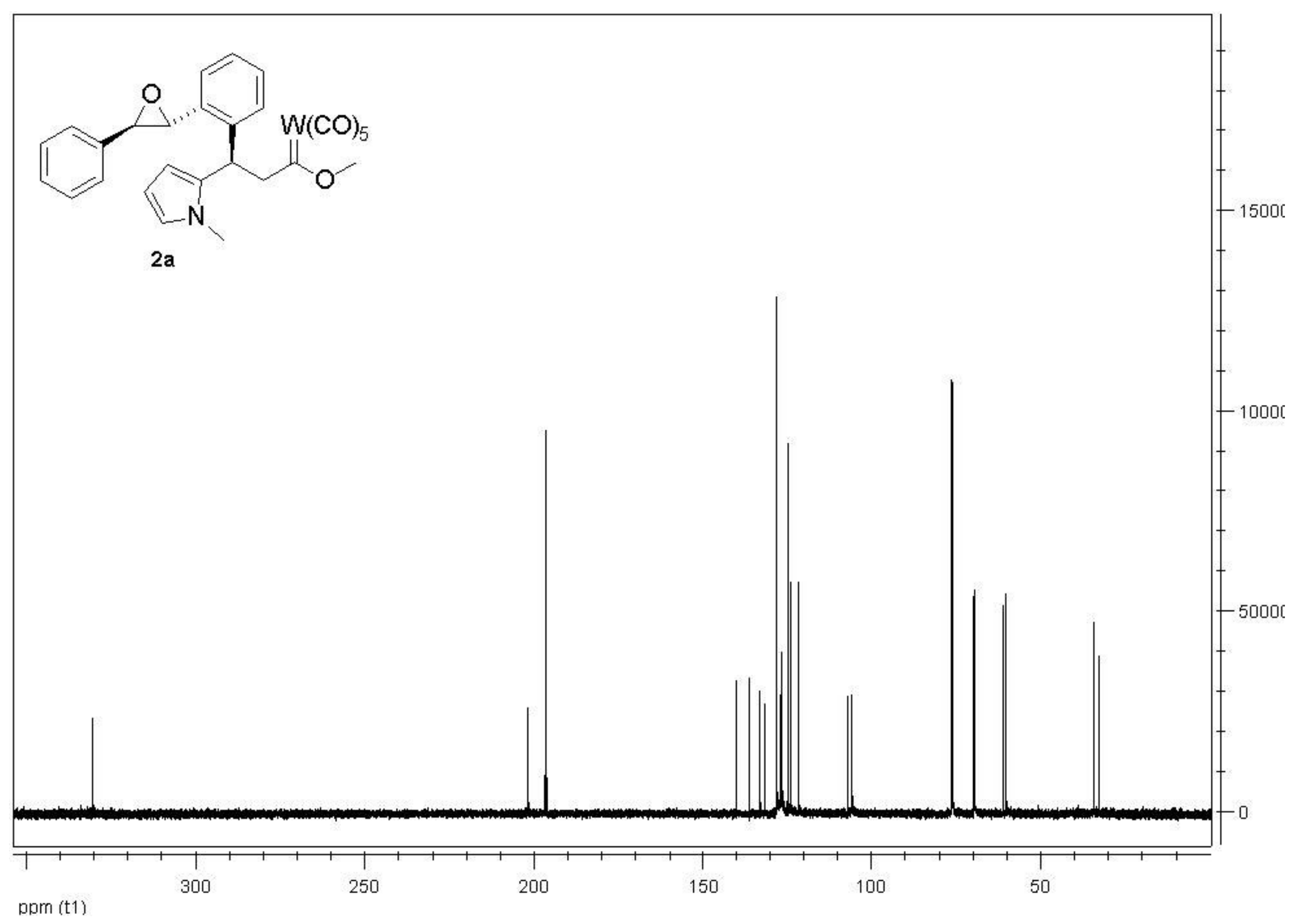




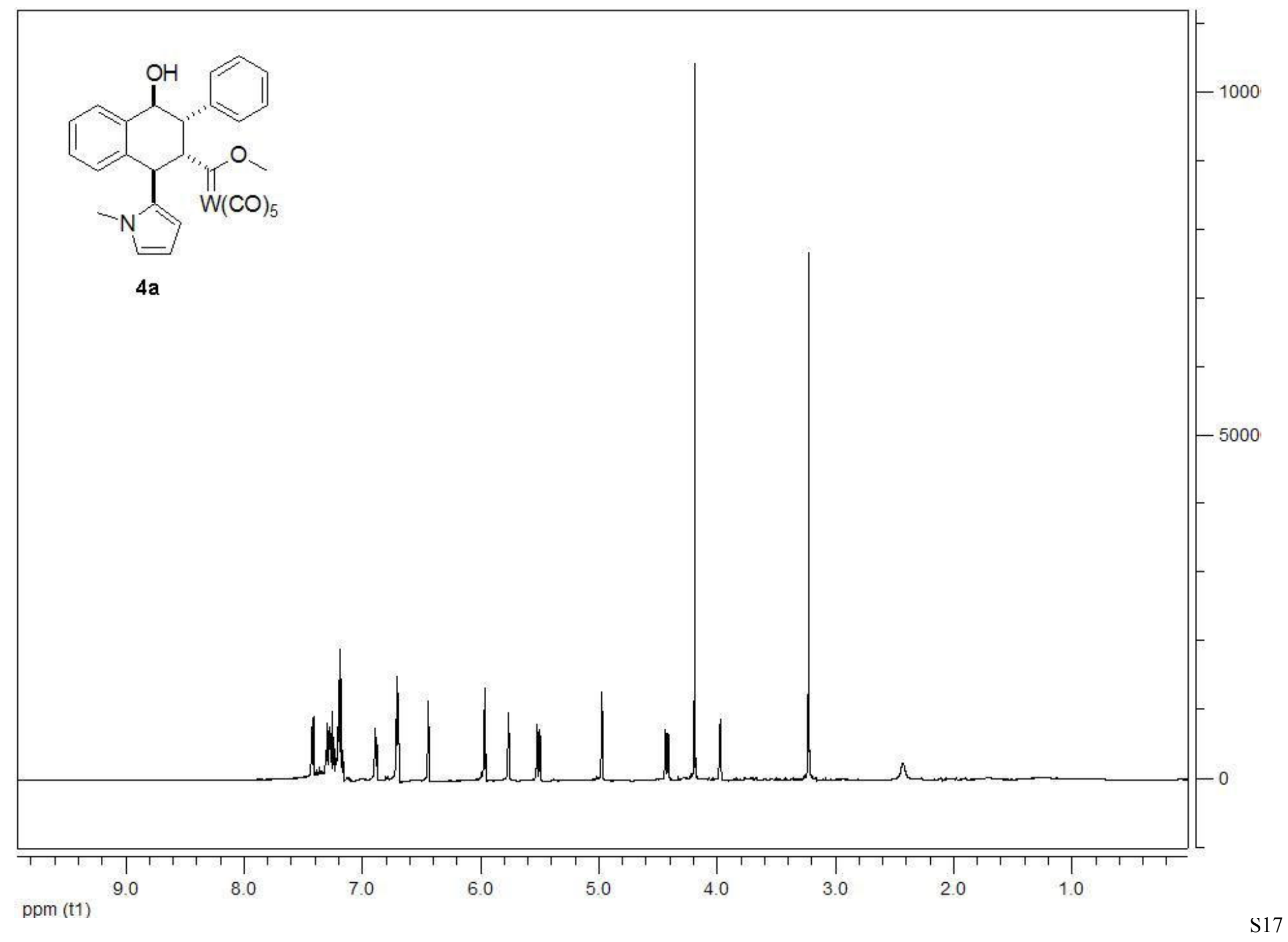




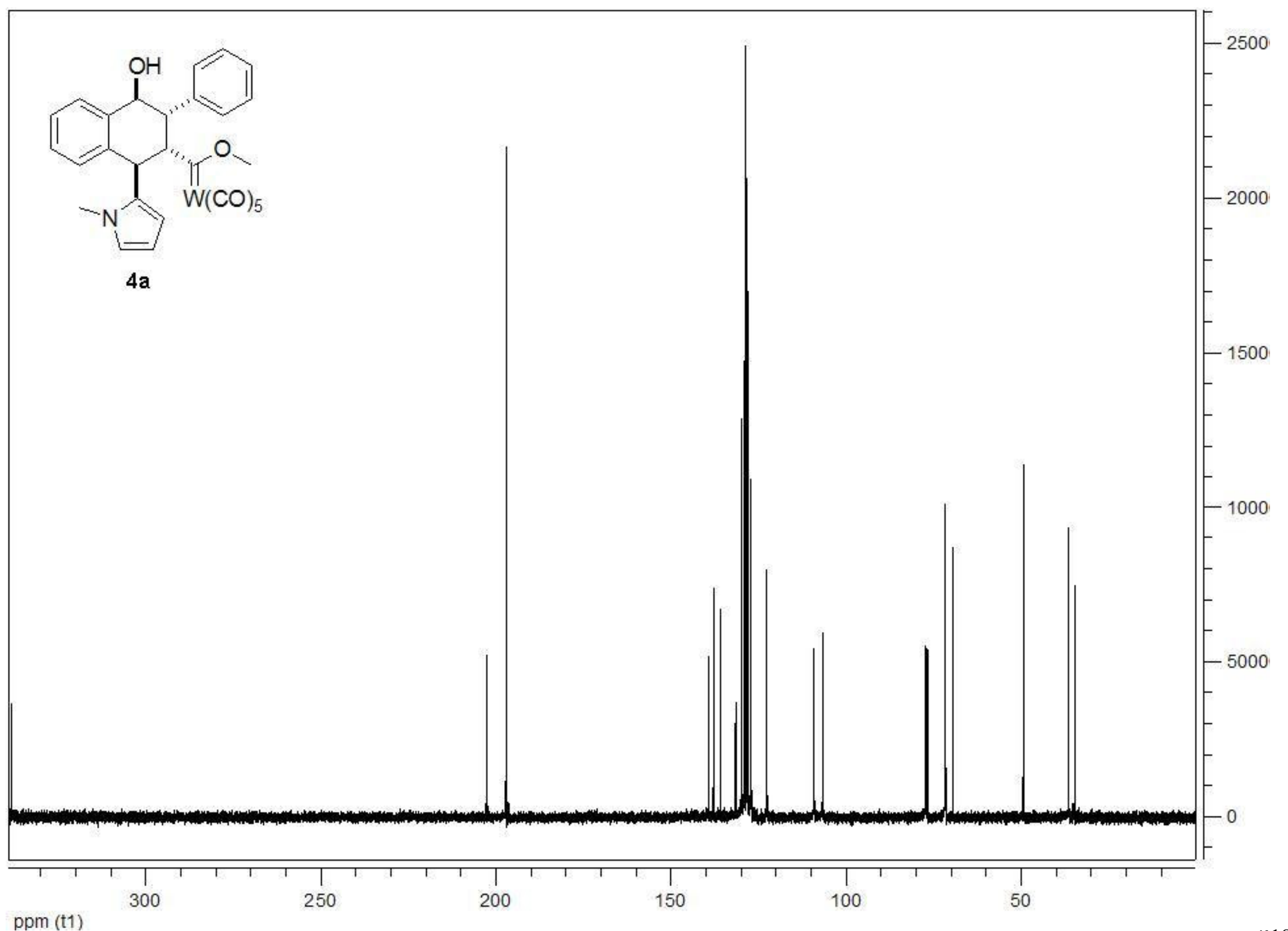




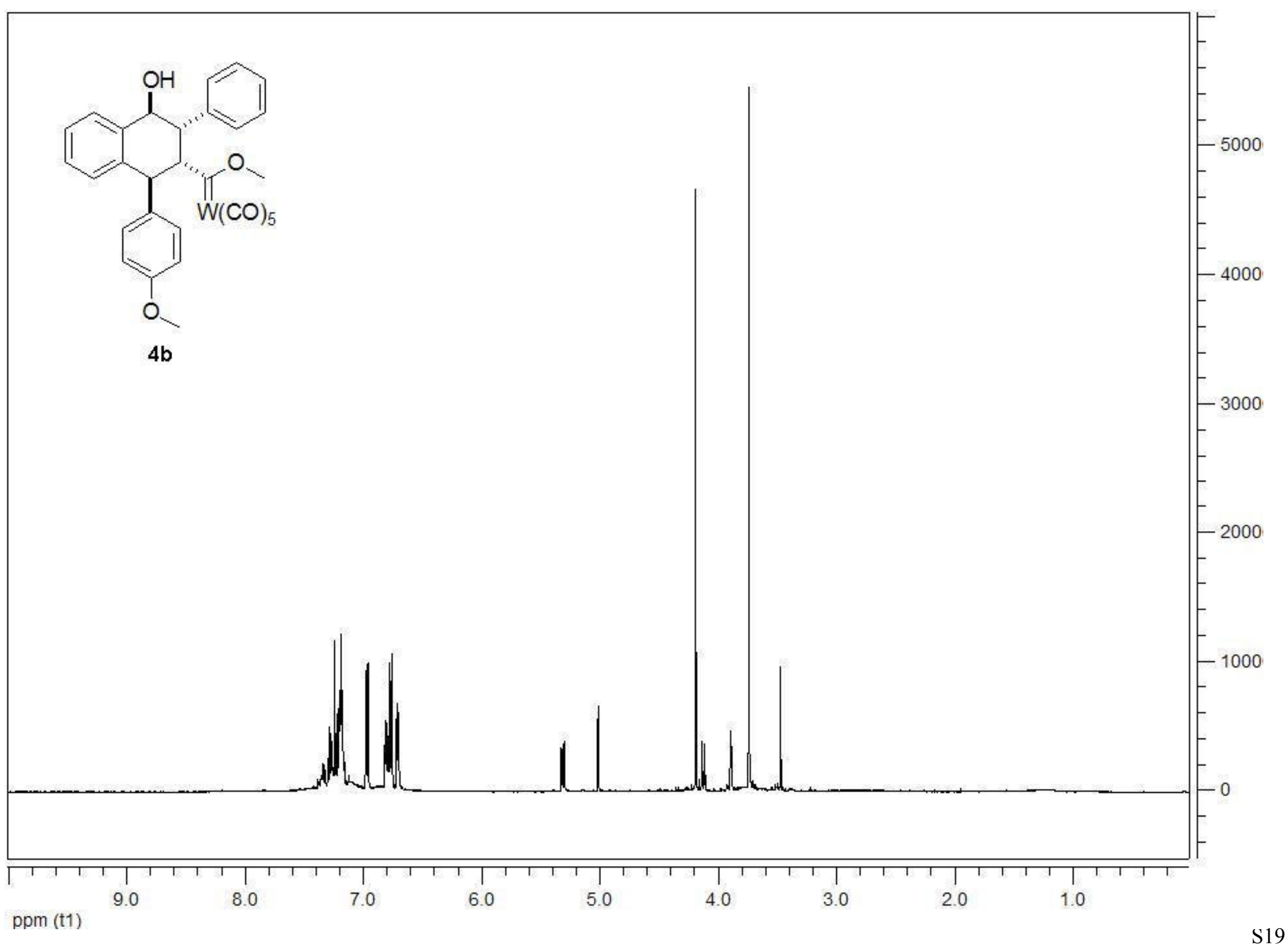




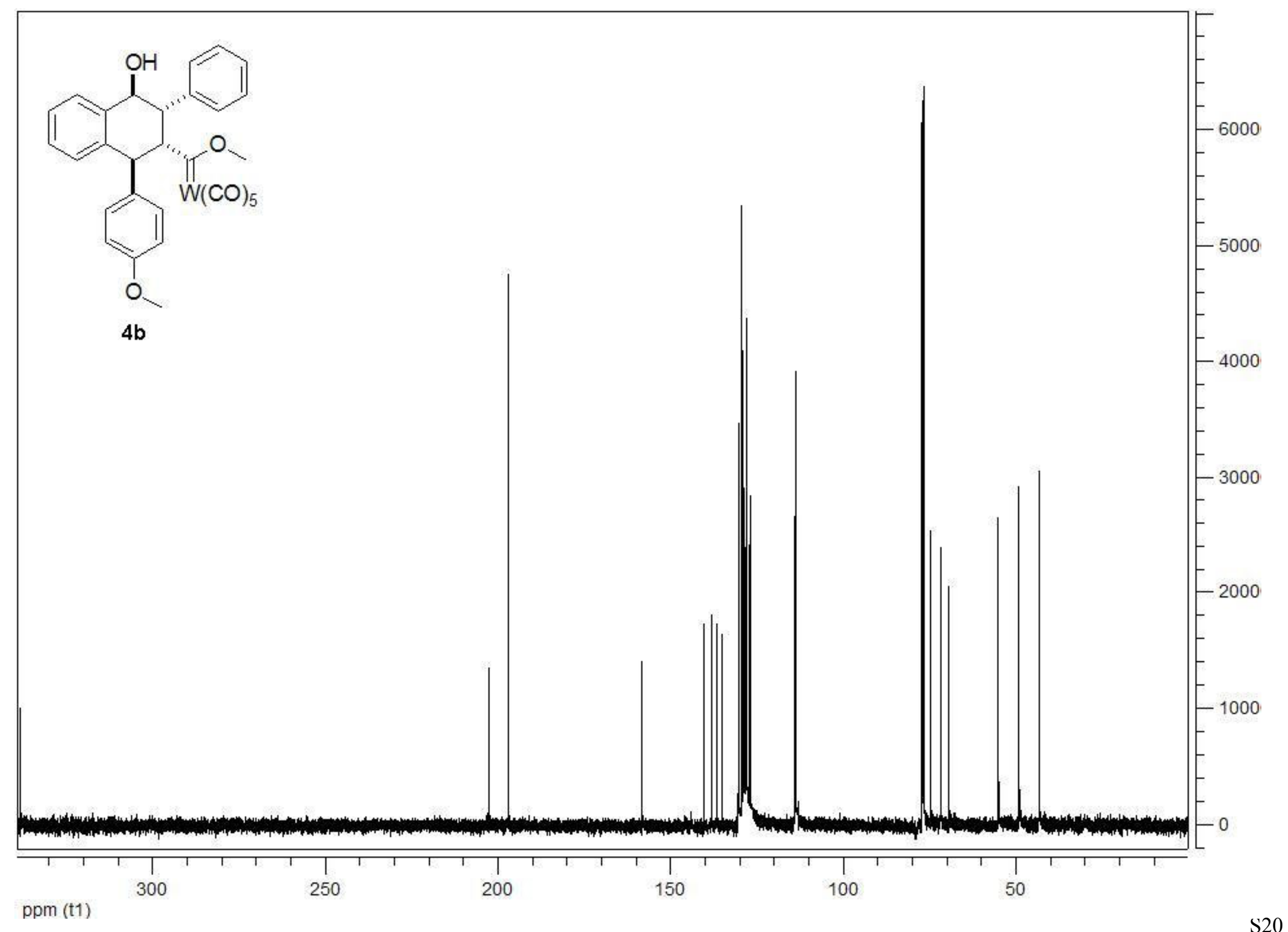




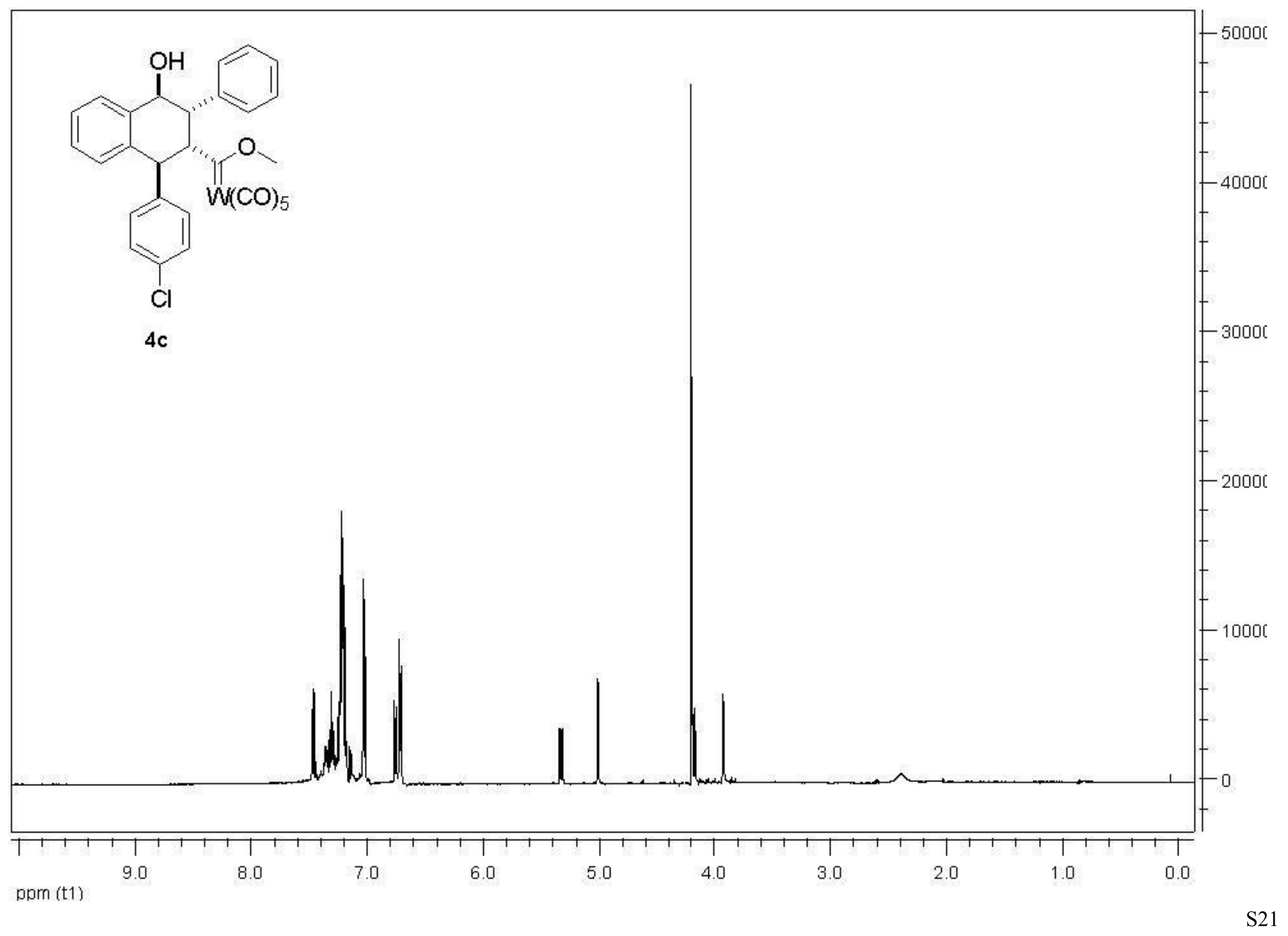




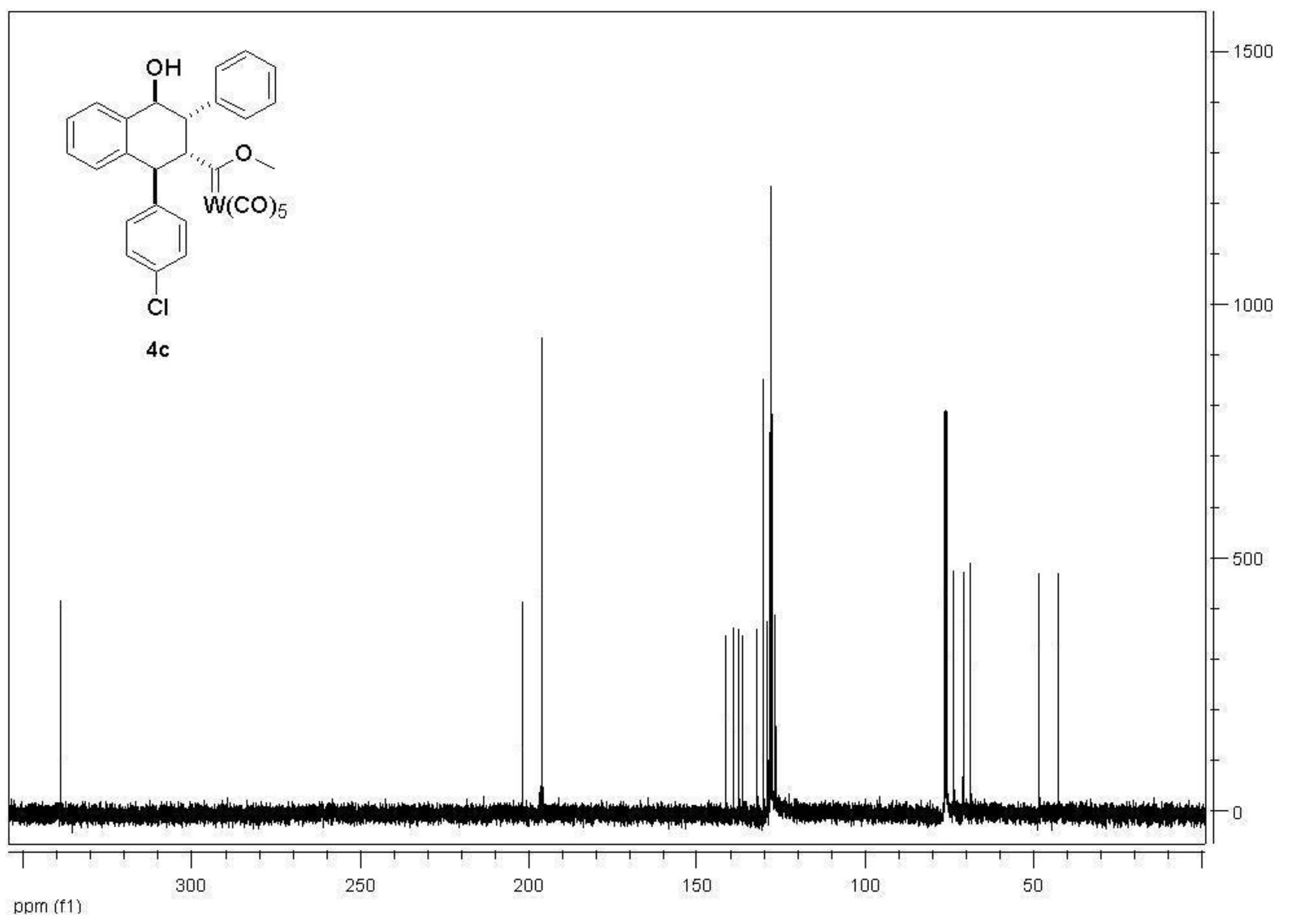




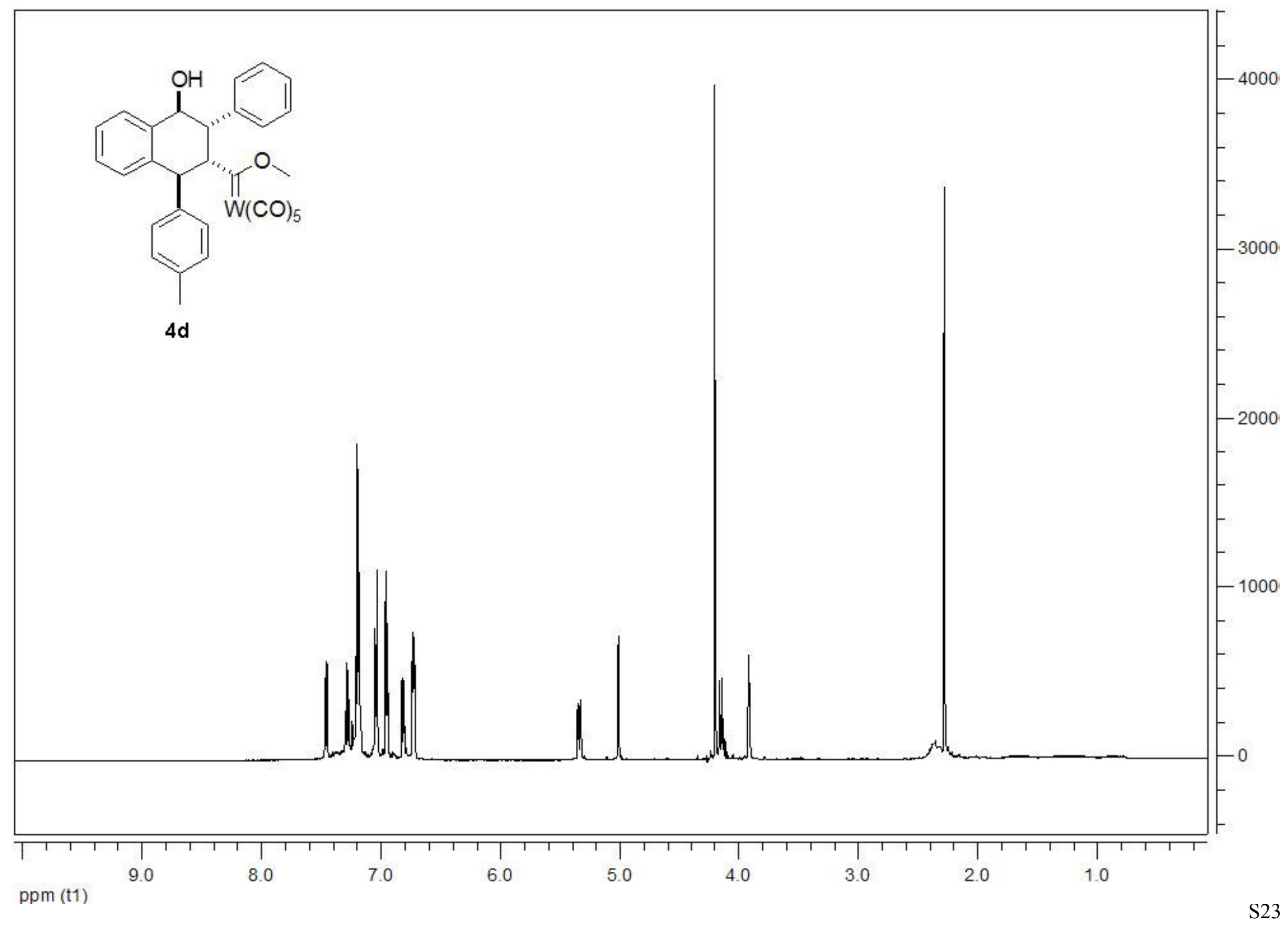




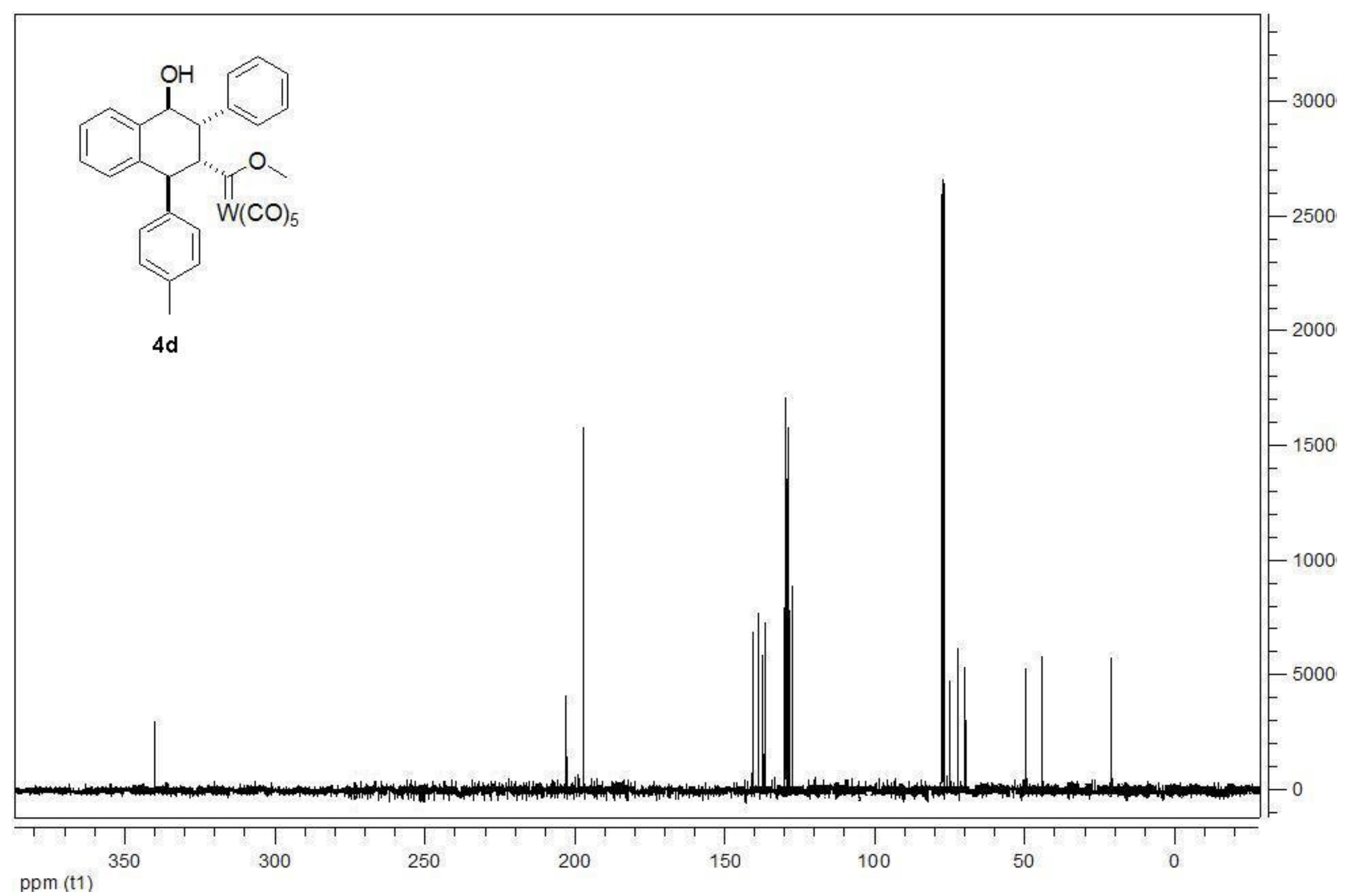




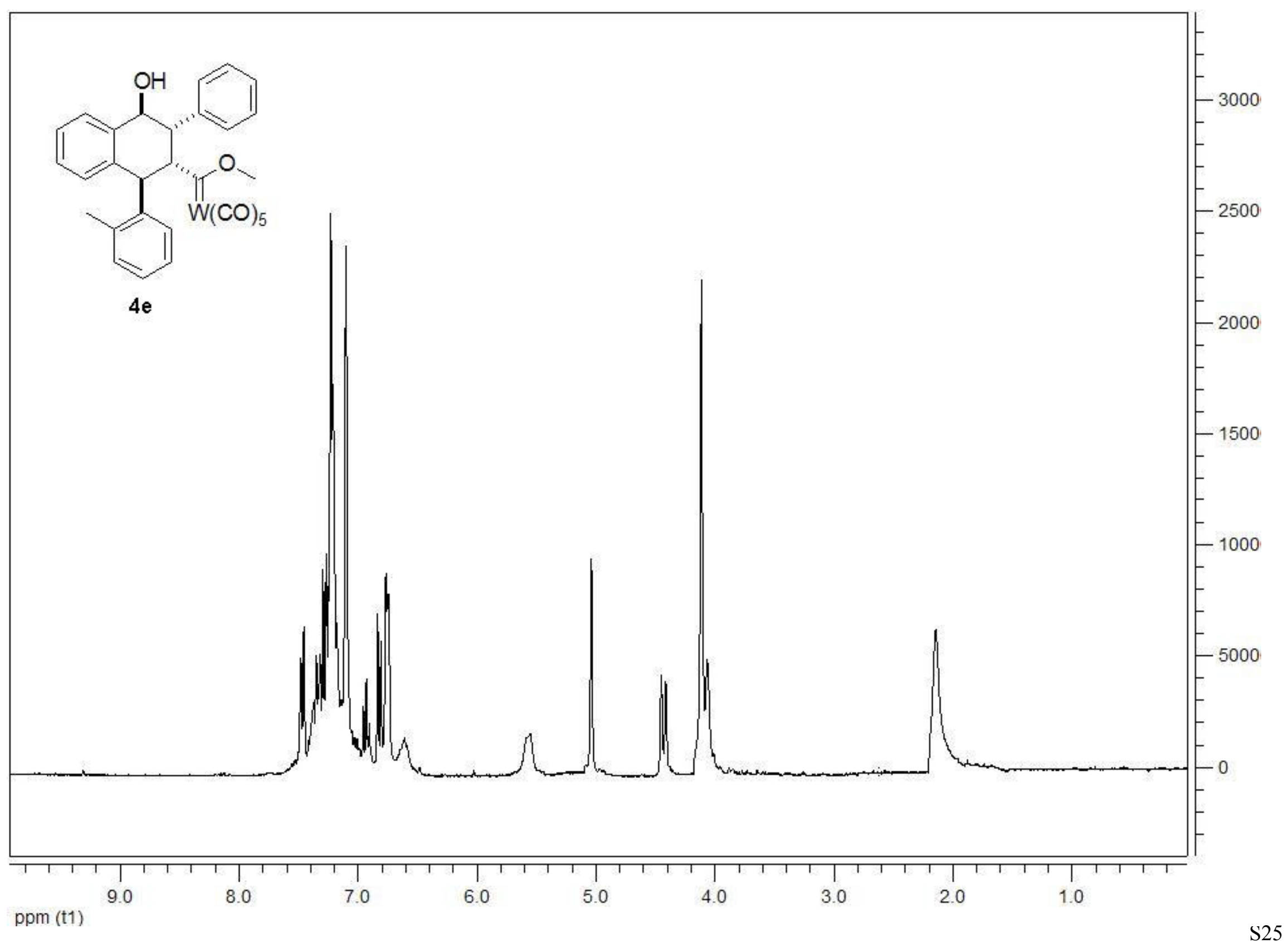




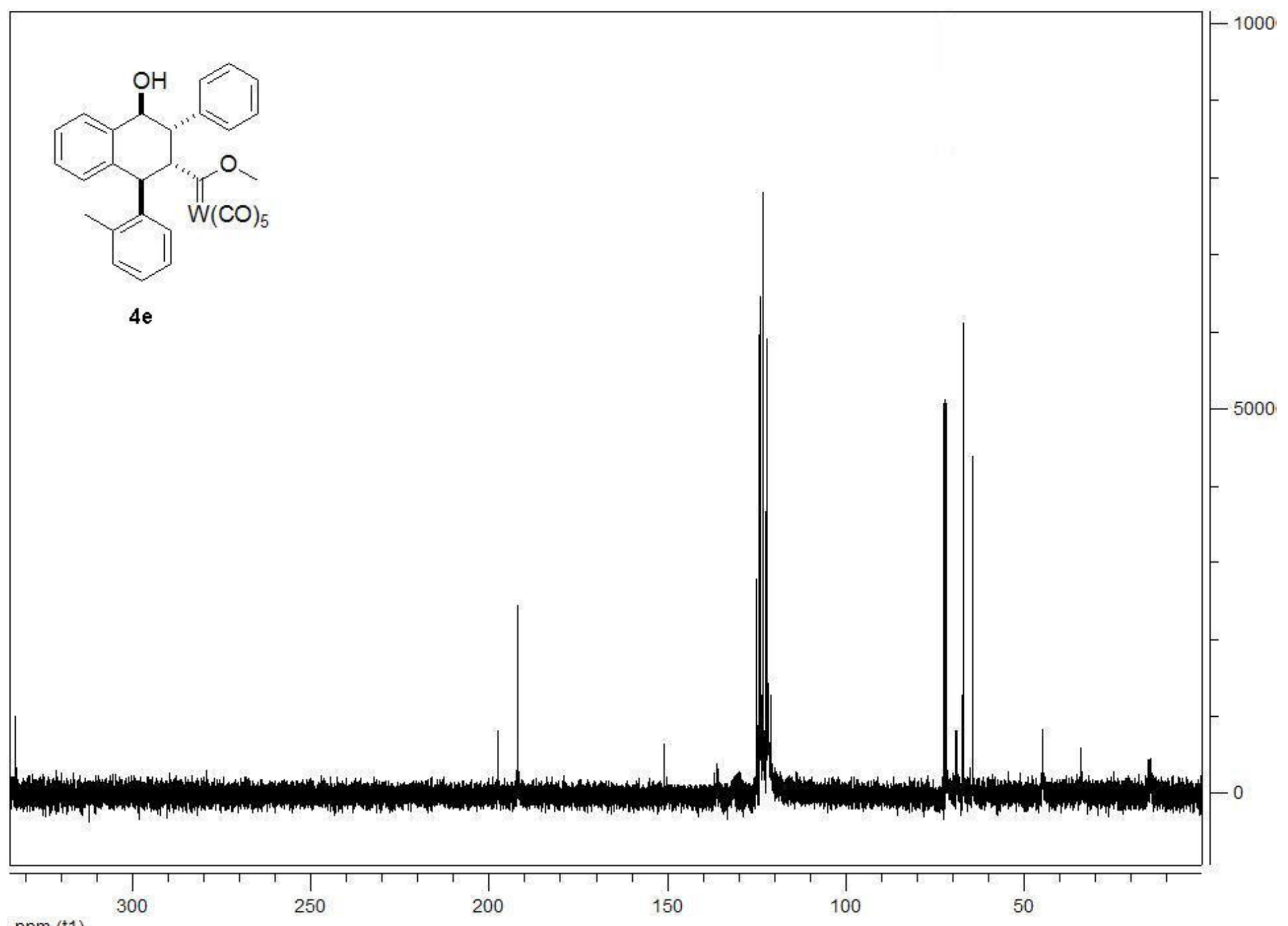




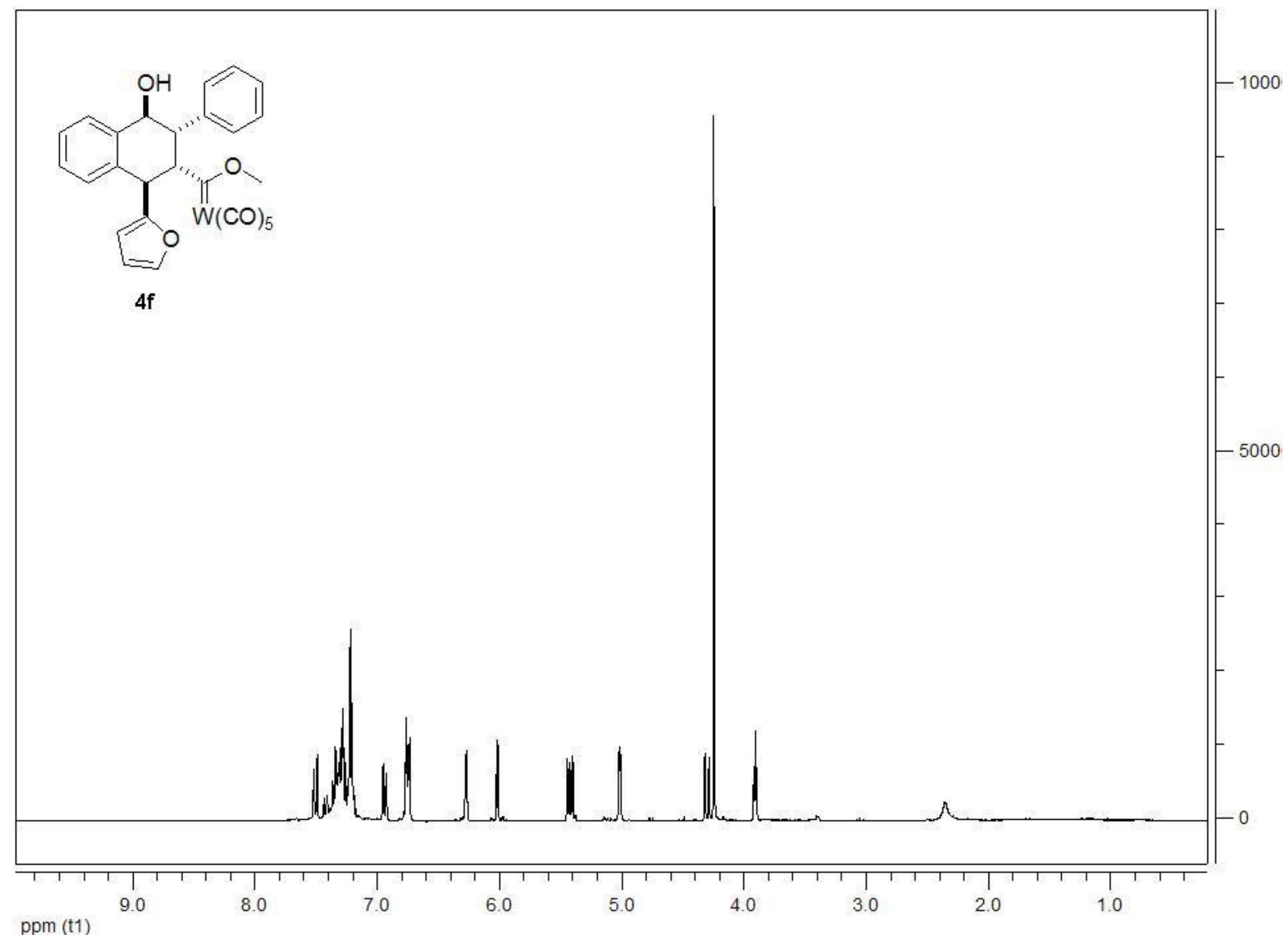

S27 


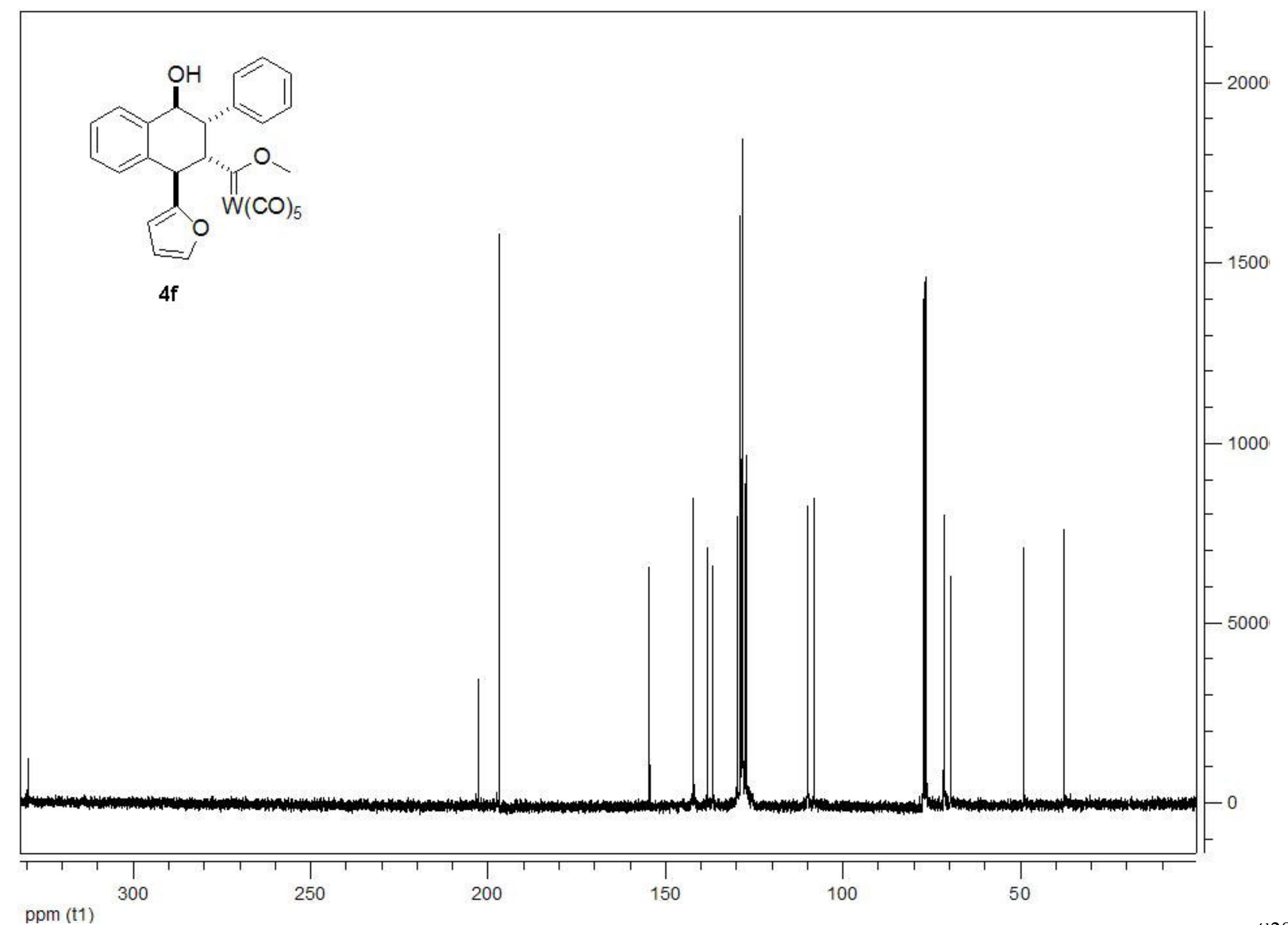




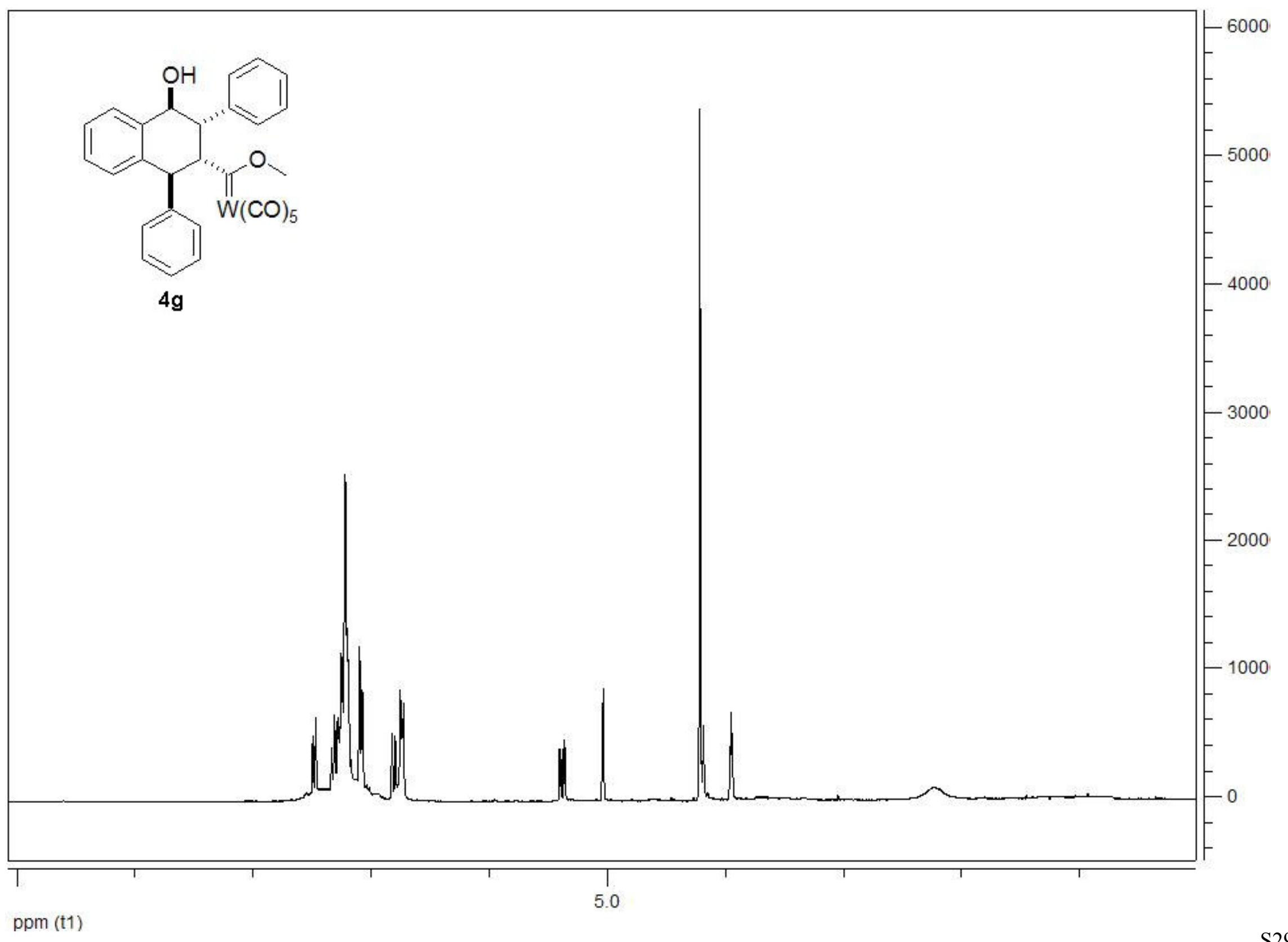




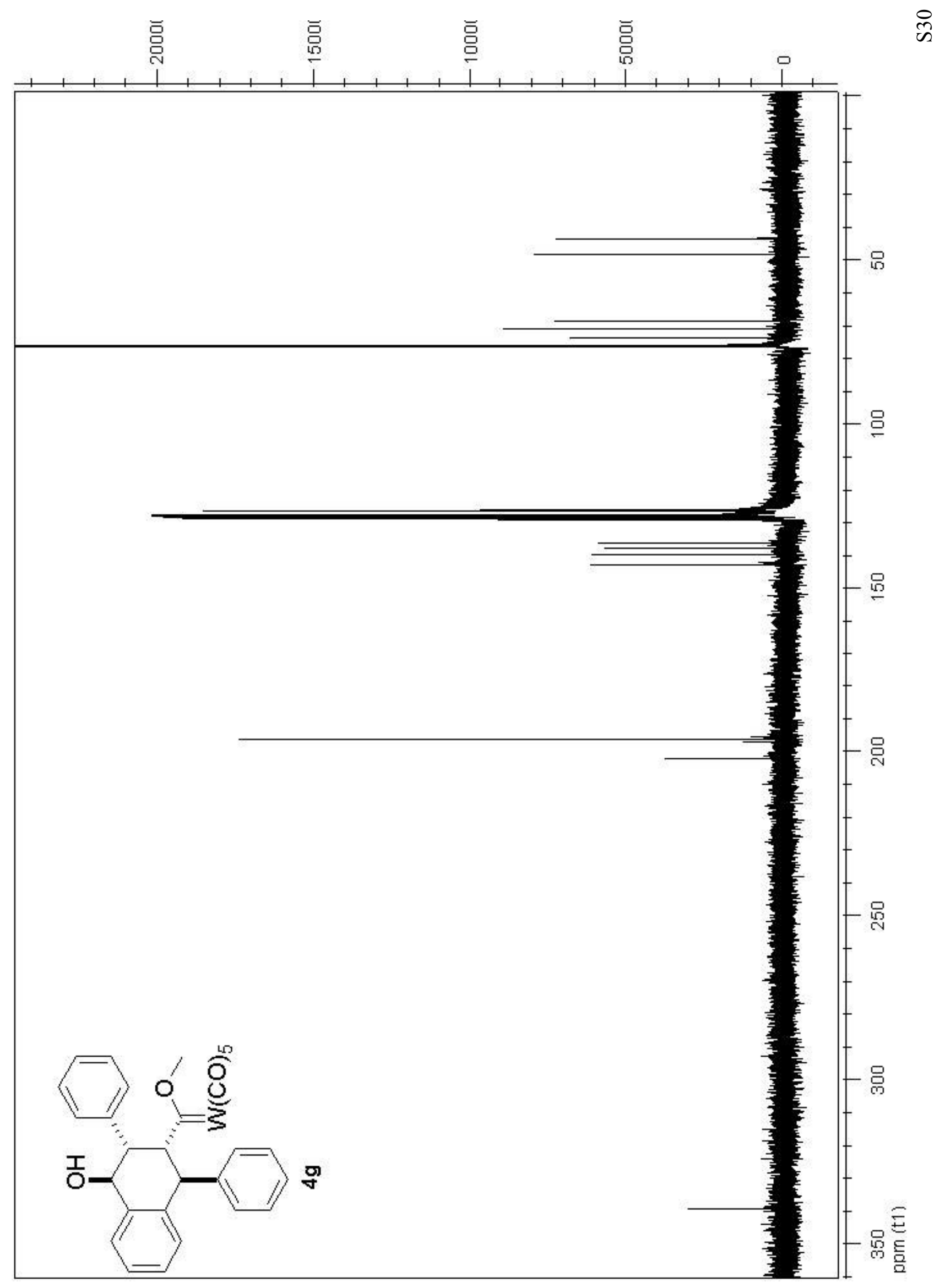




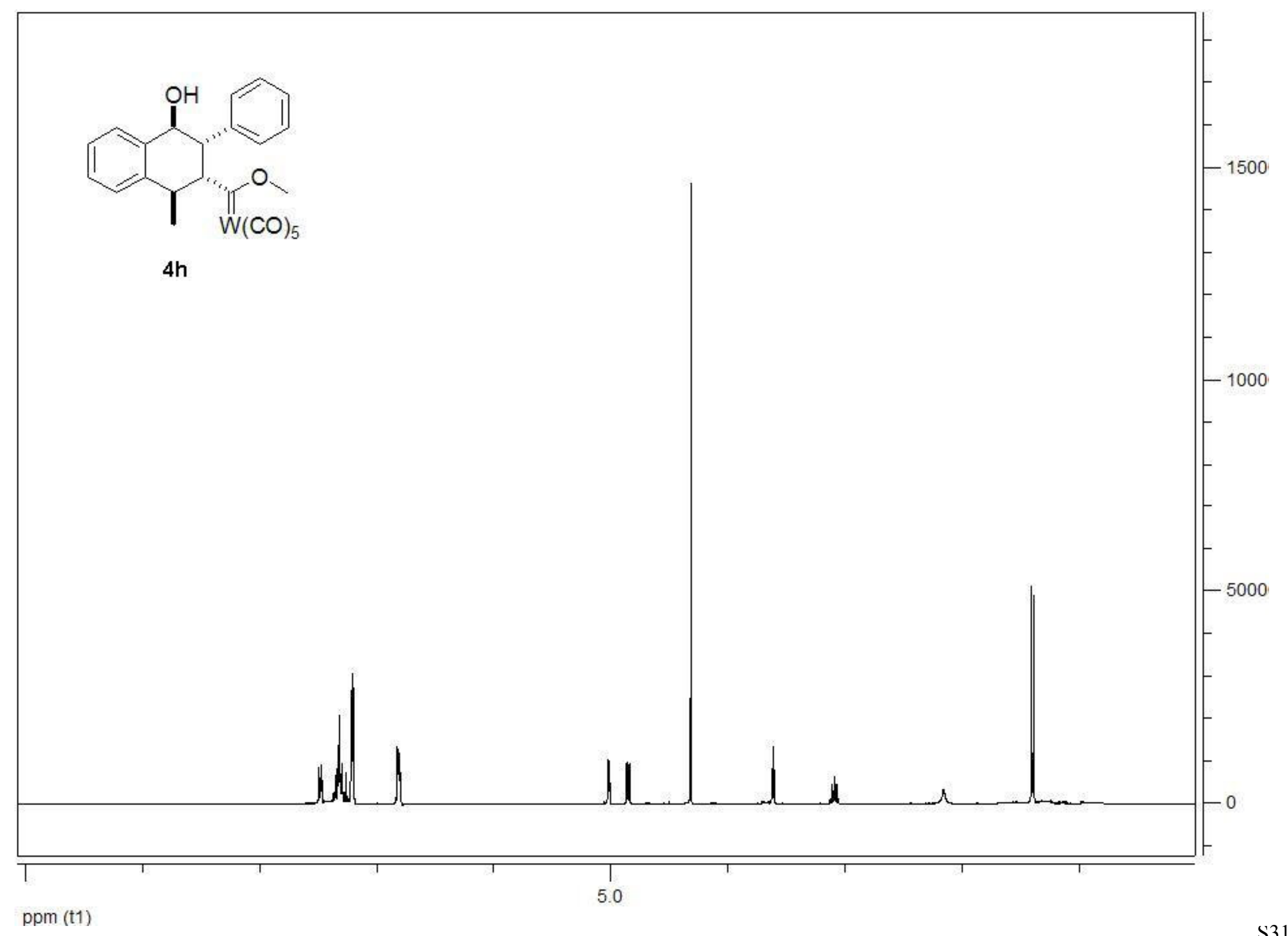




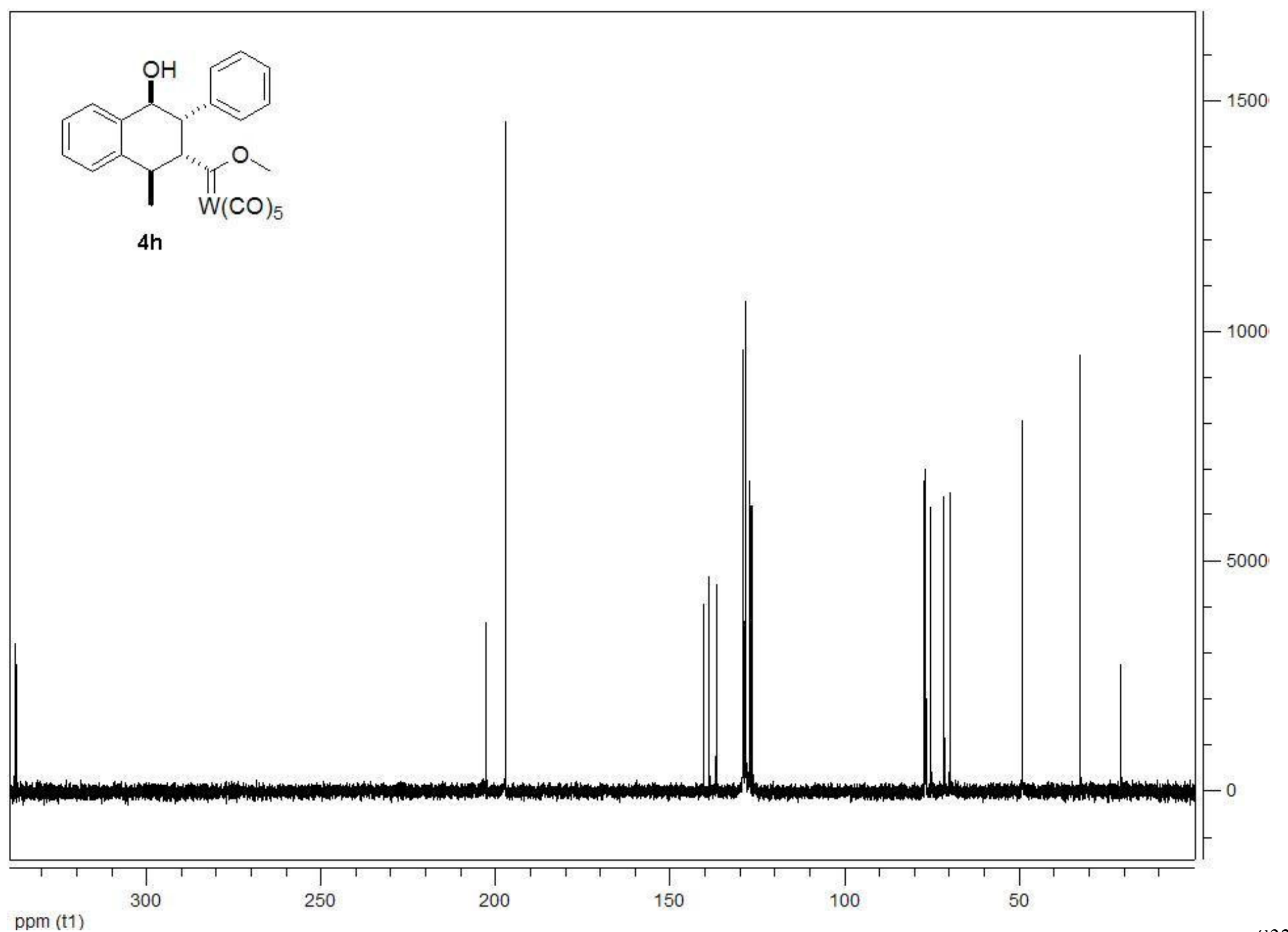




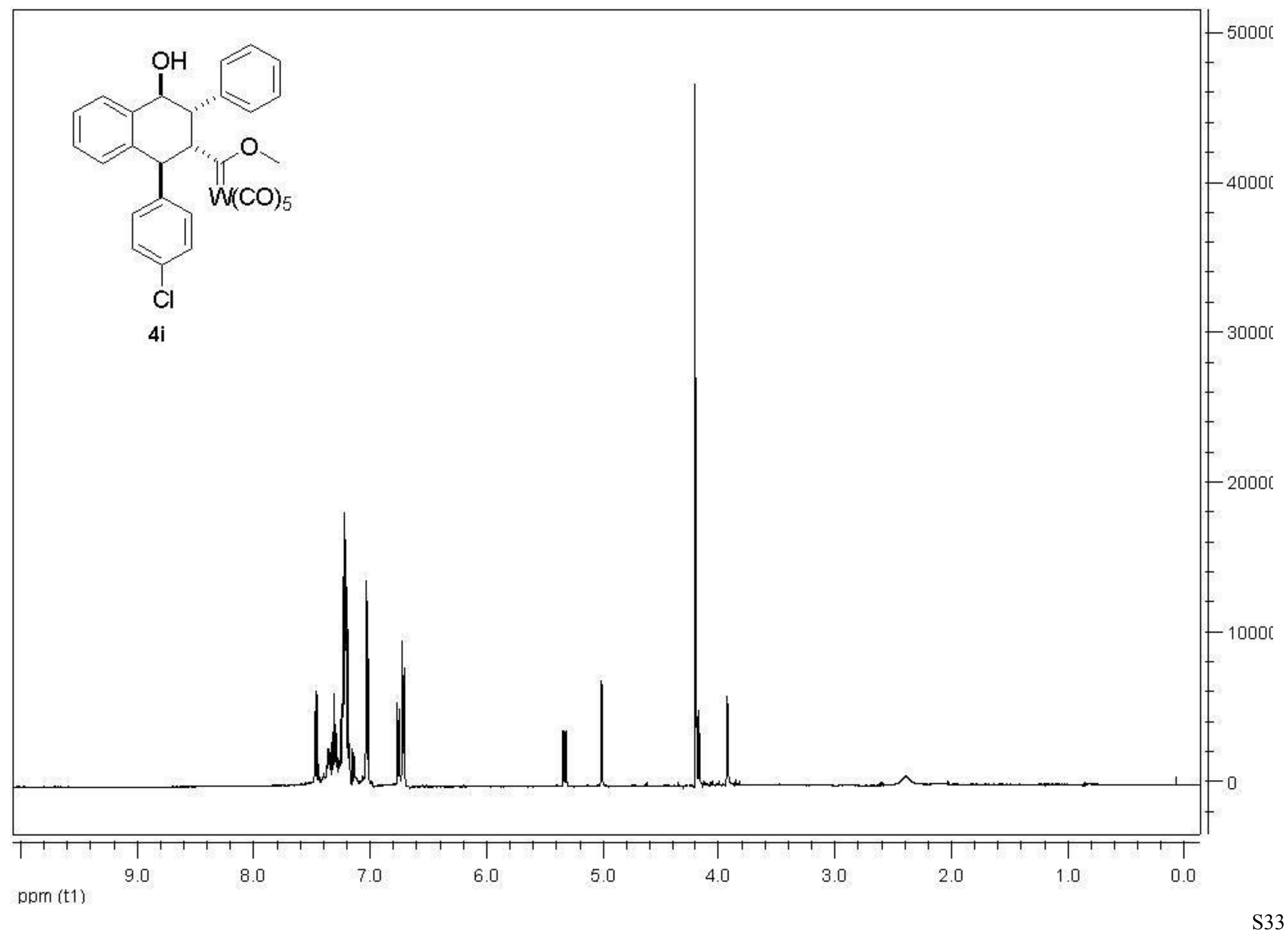




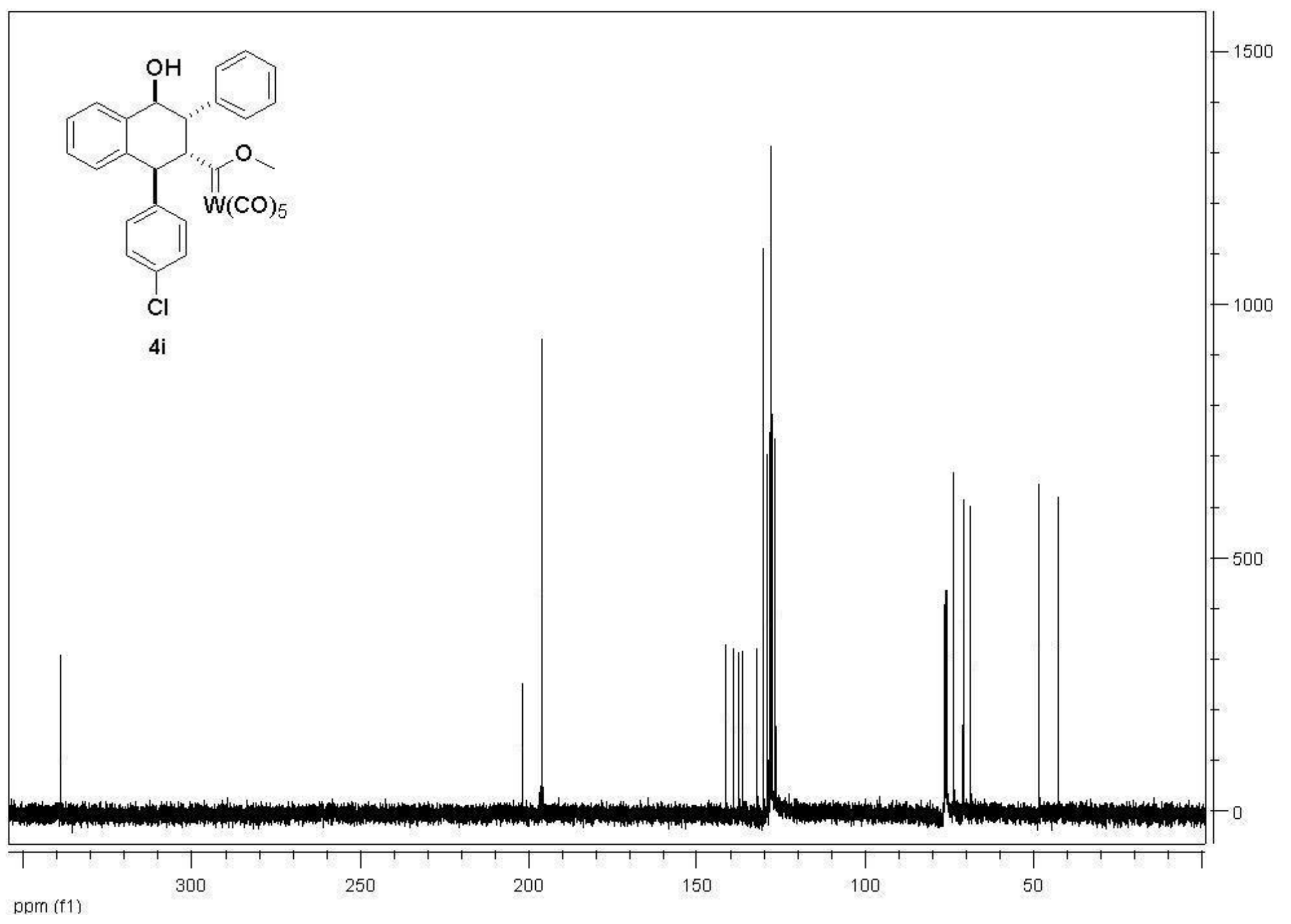




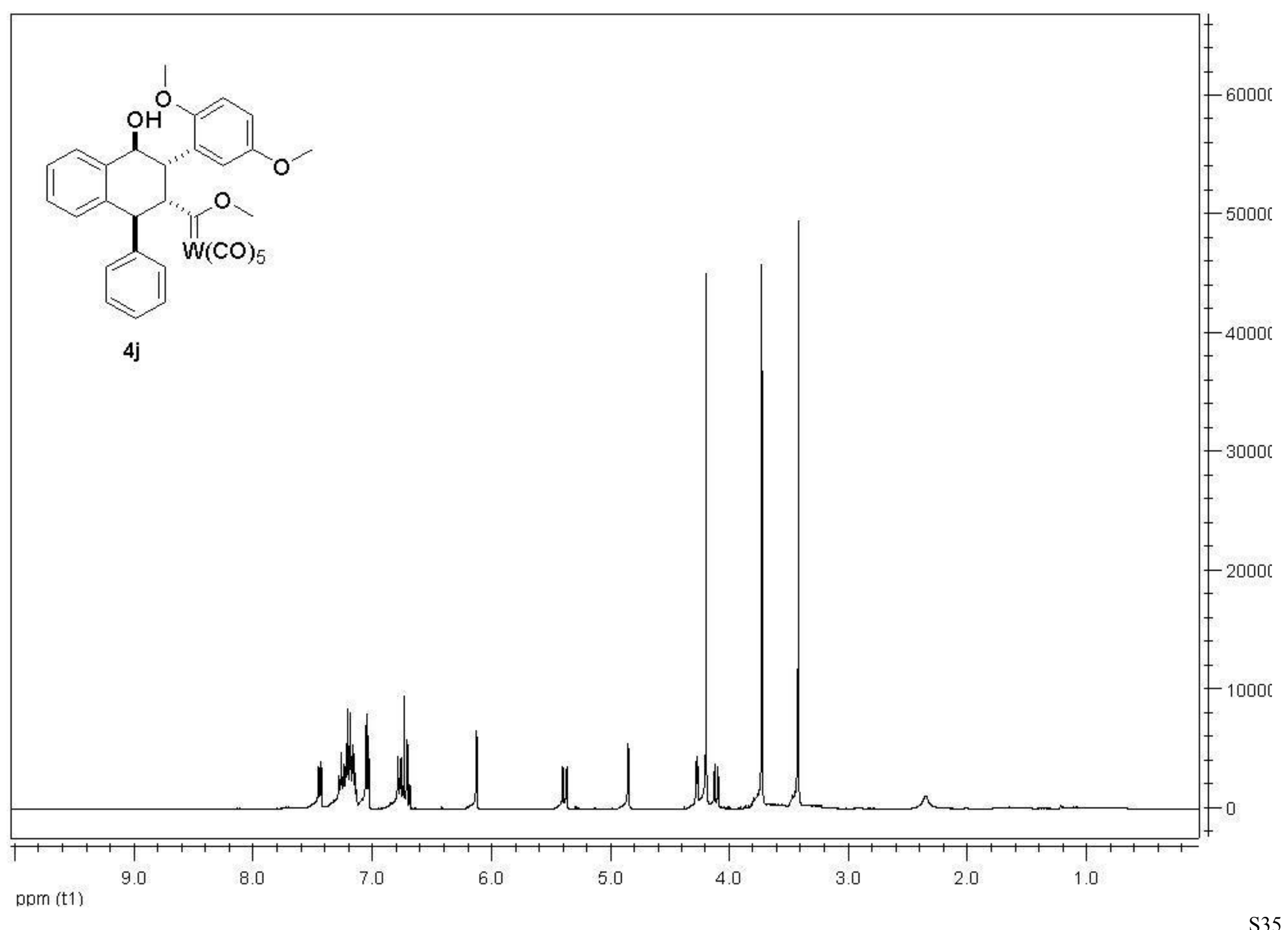




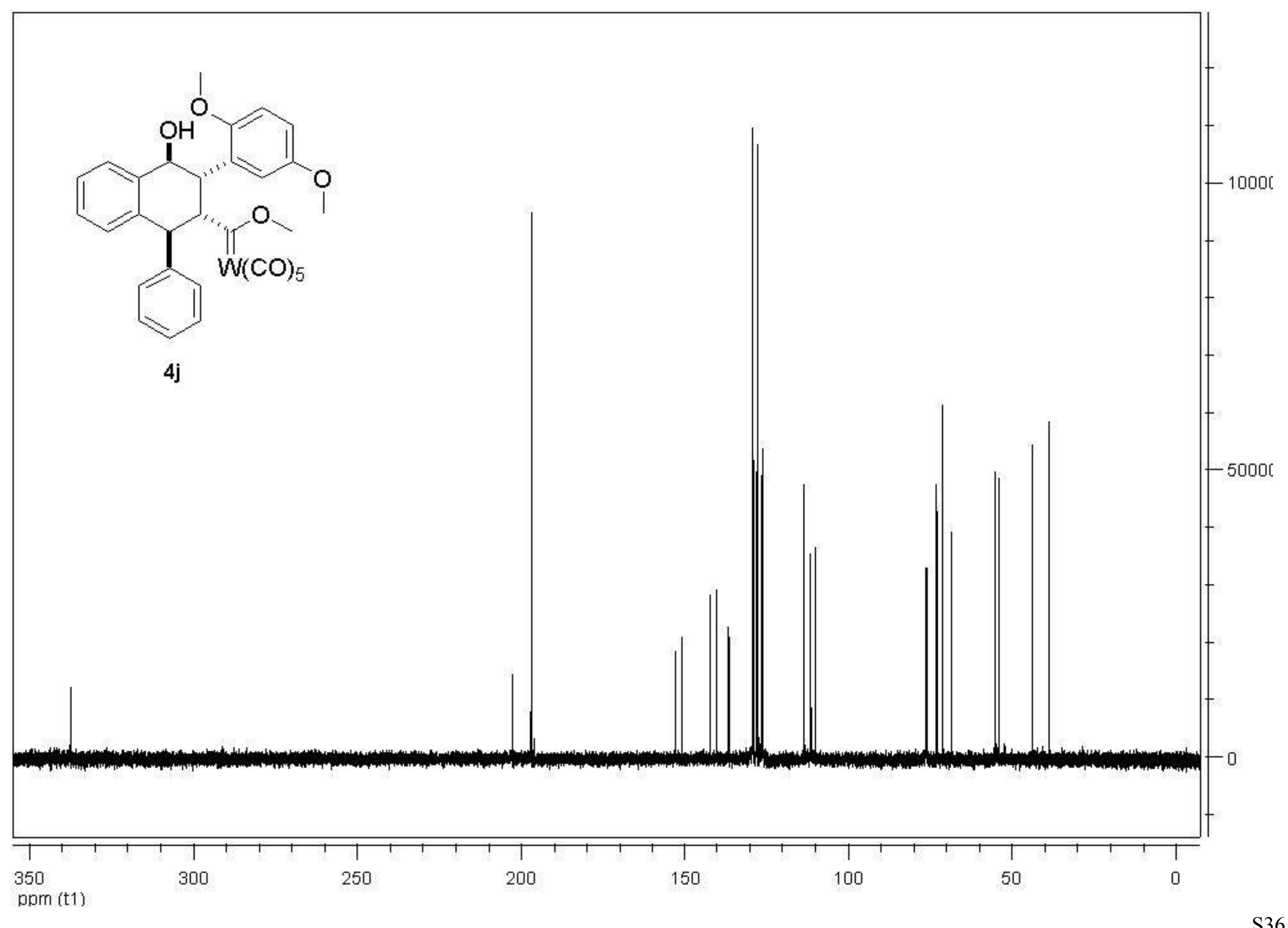




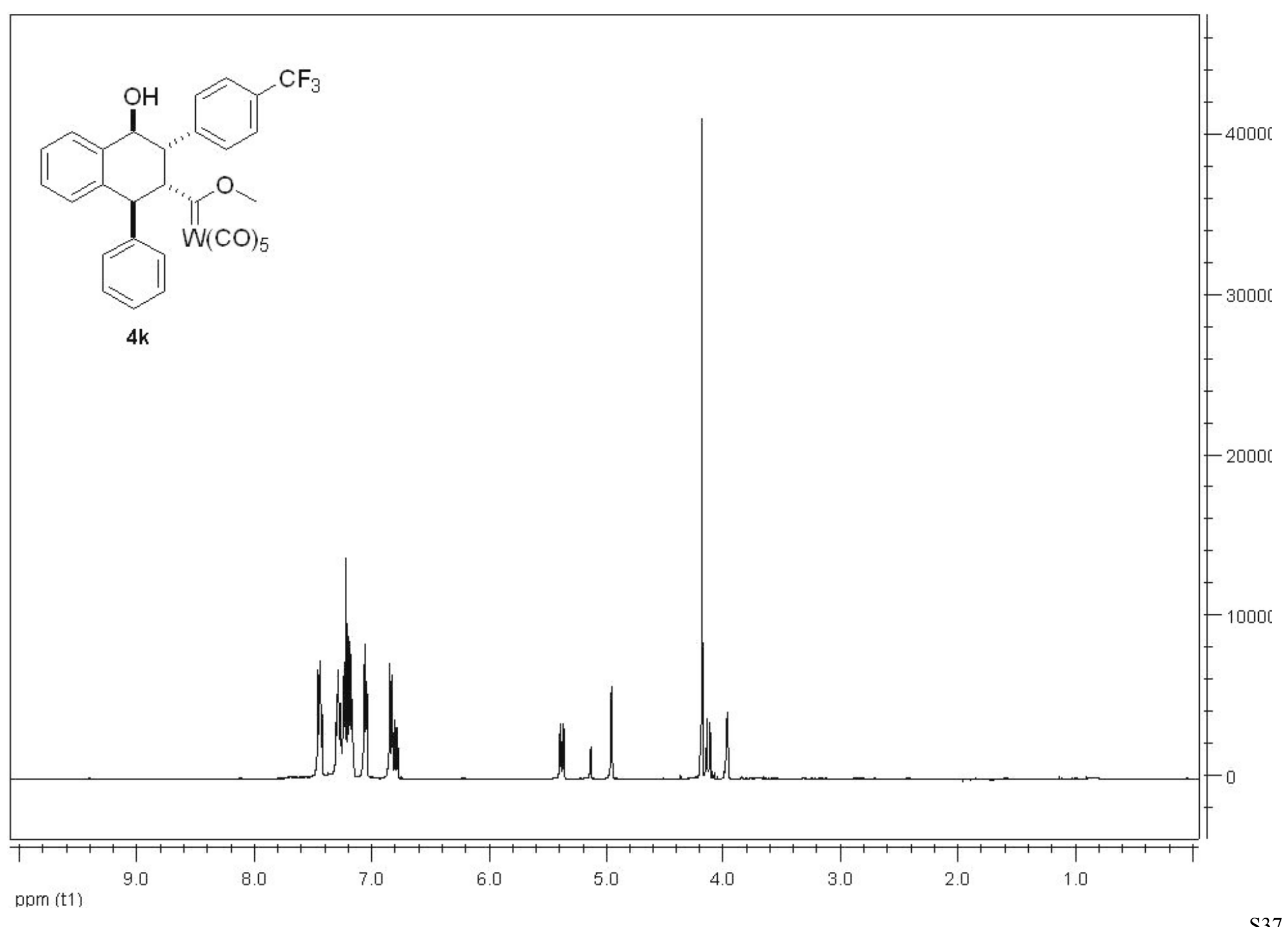




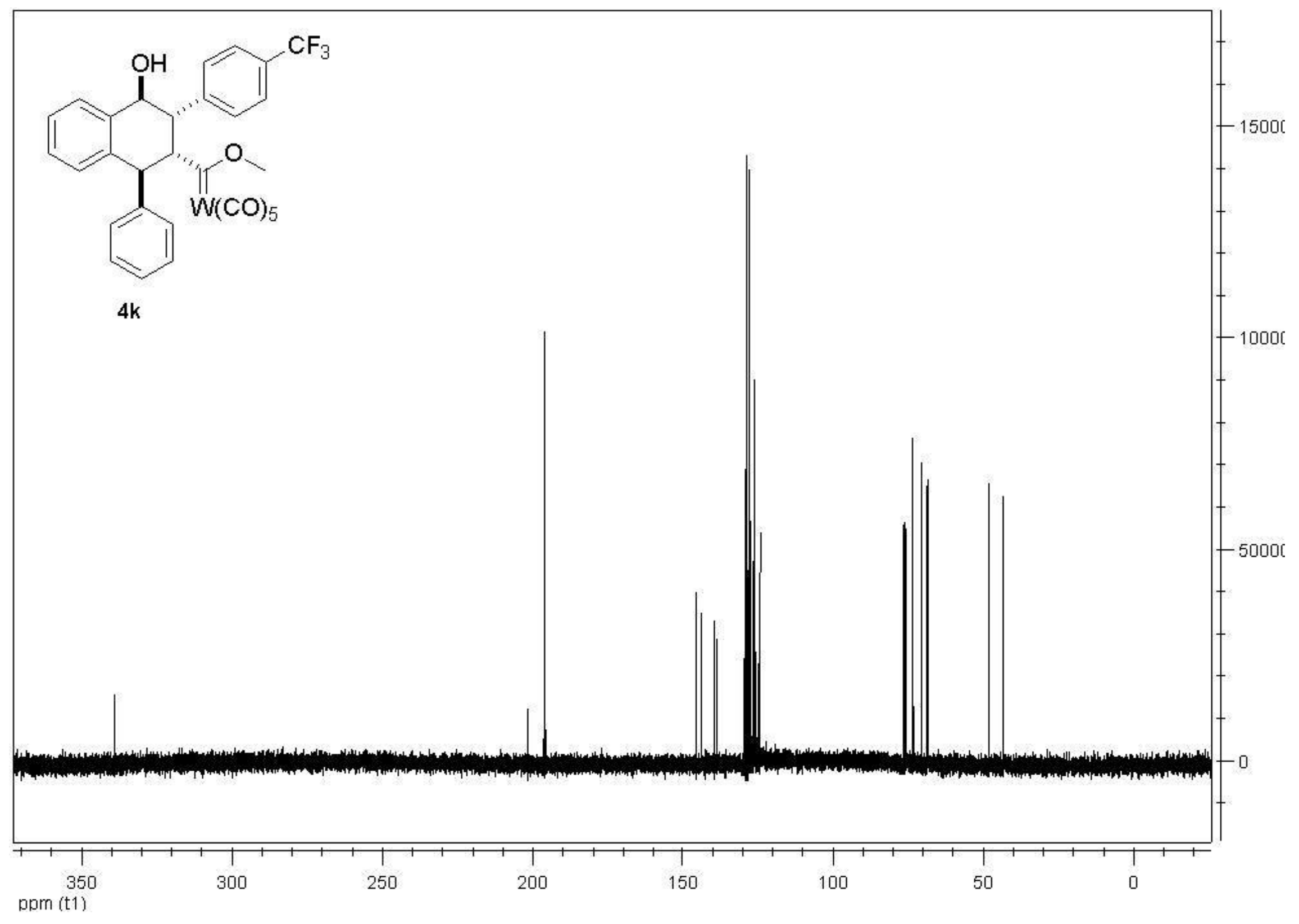




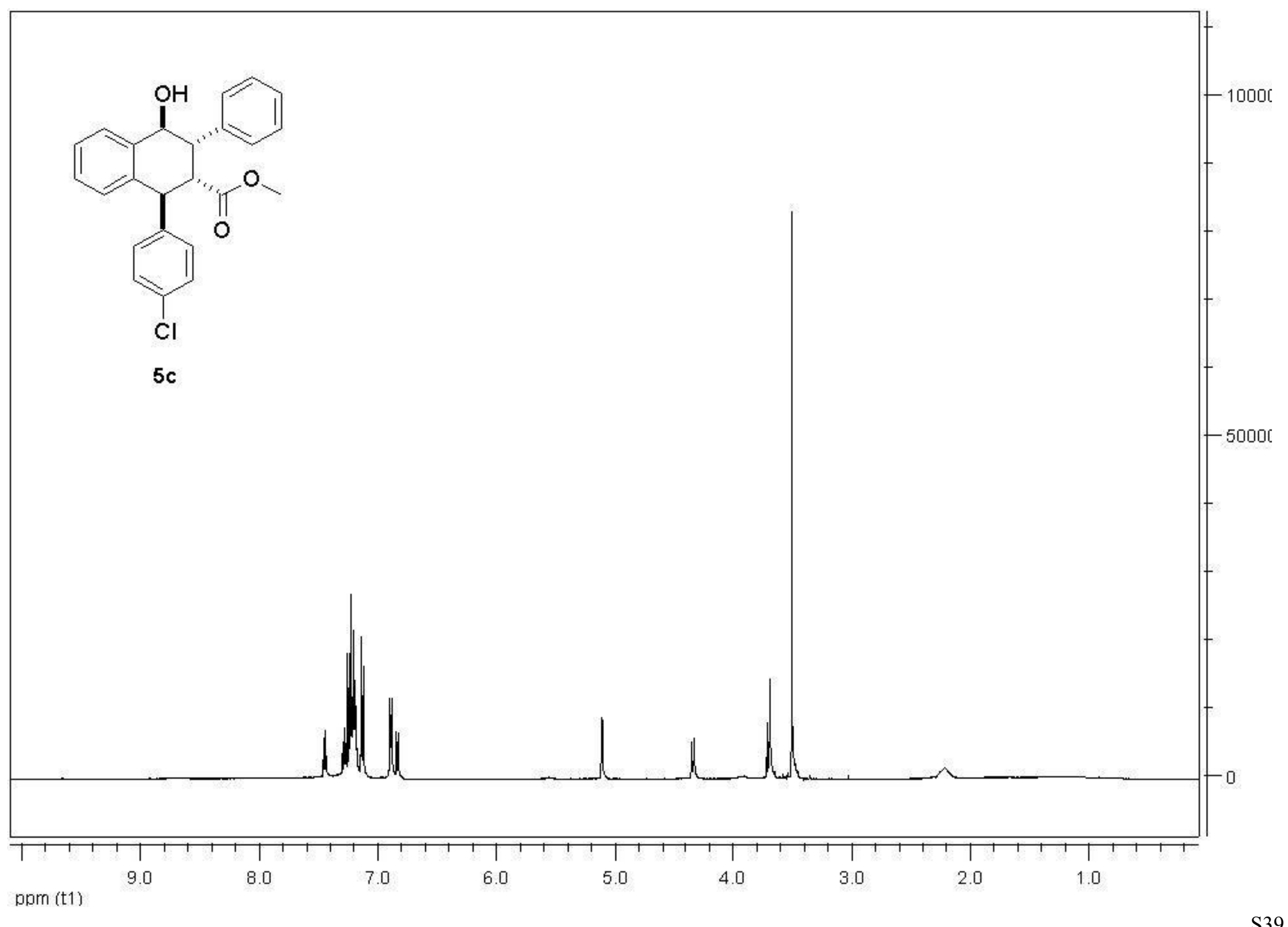




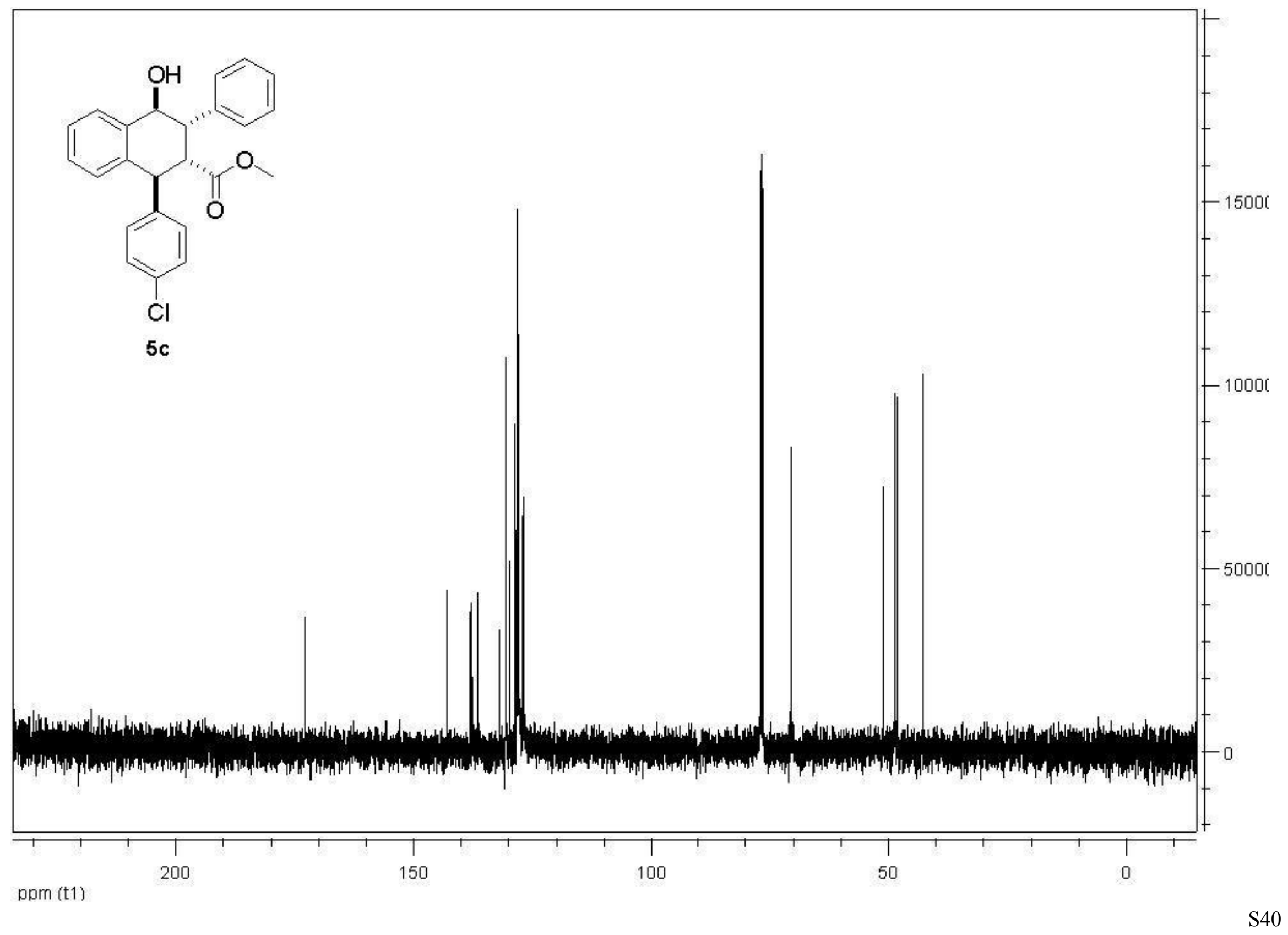




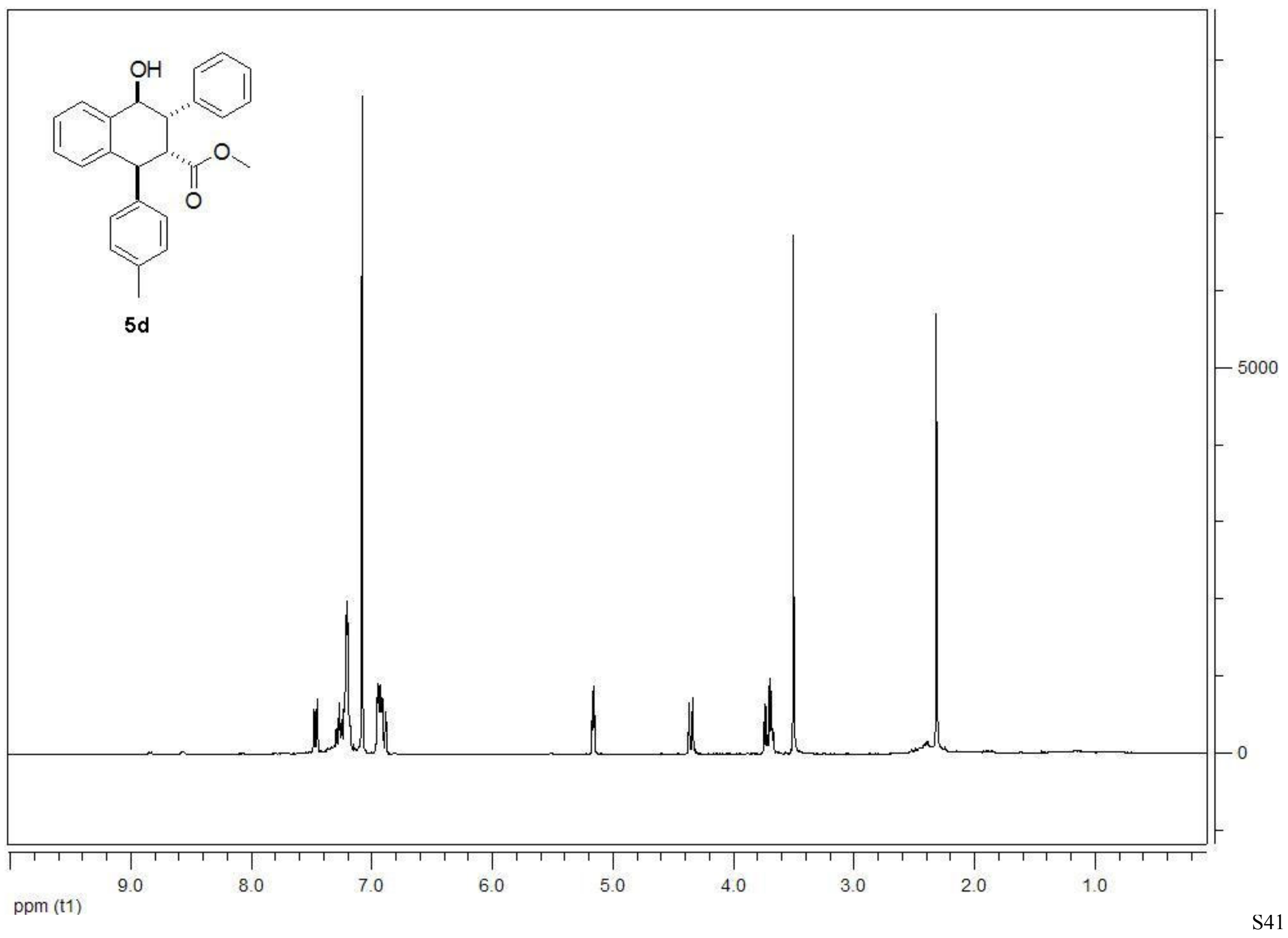




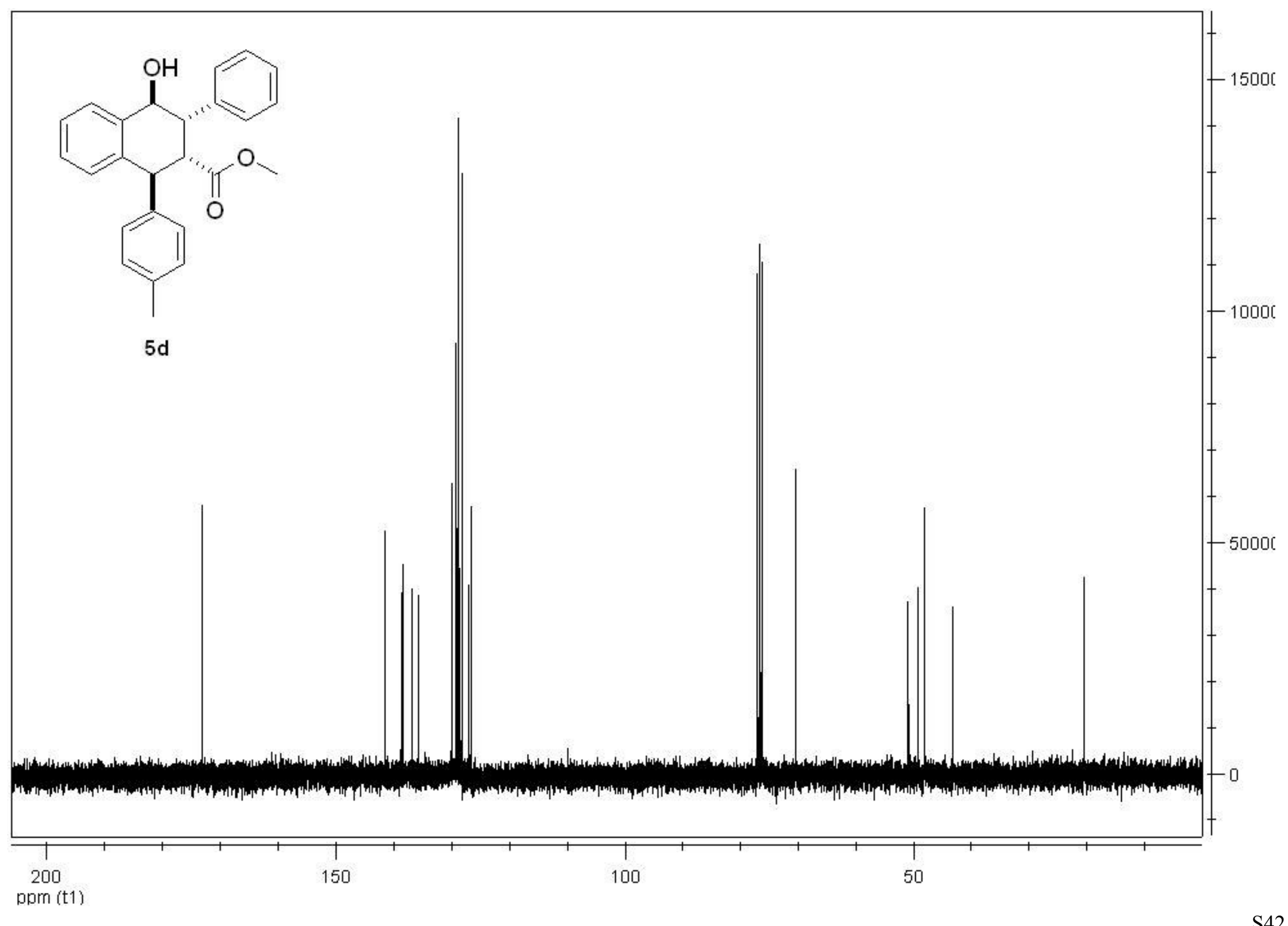




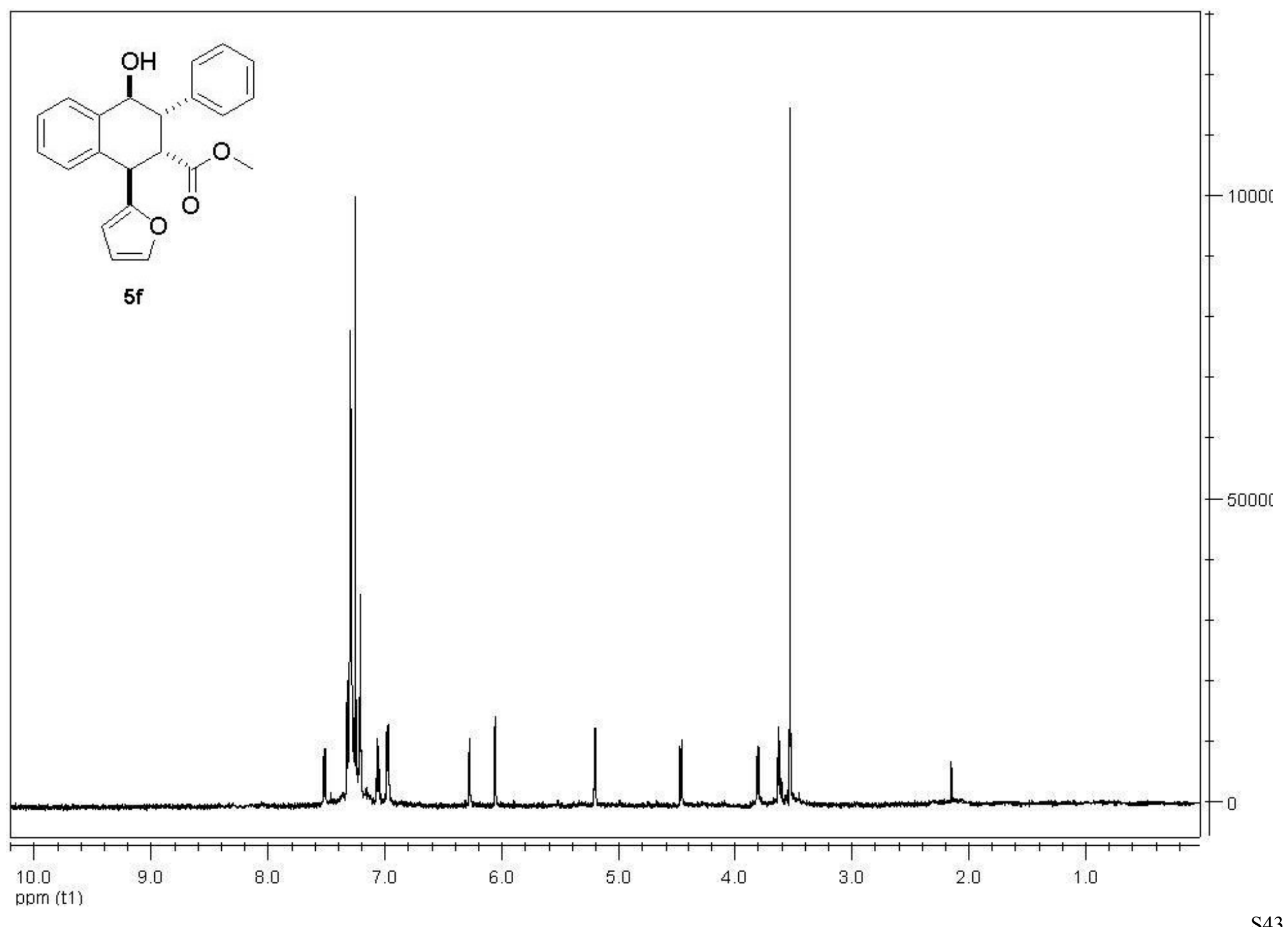




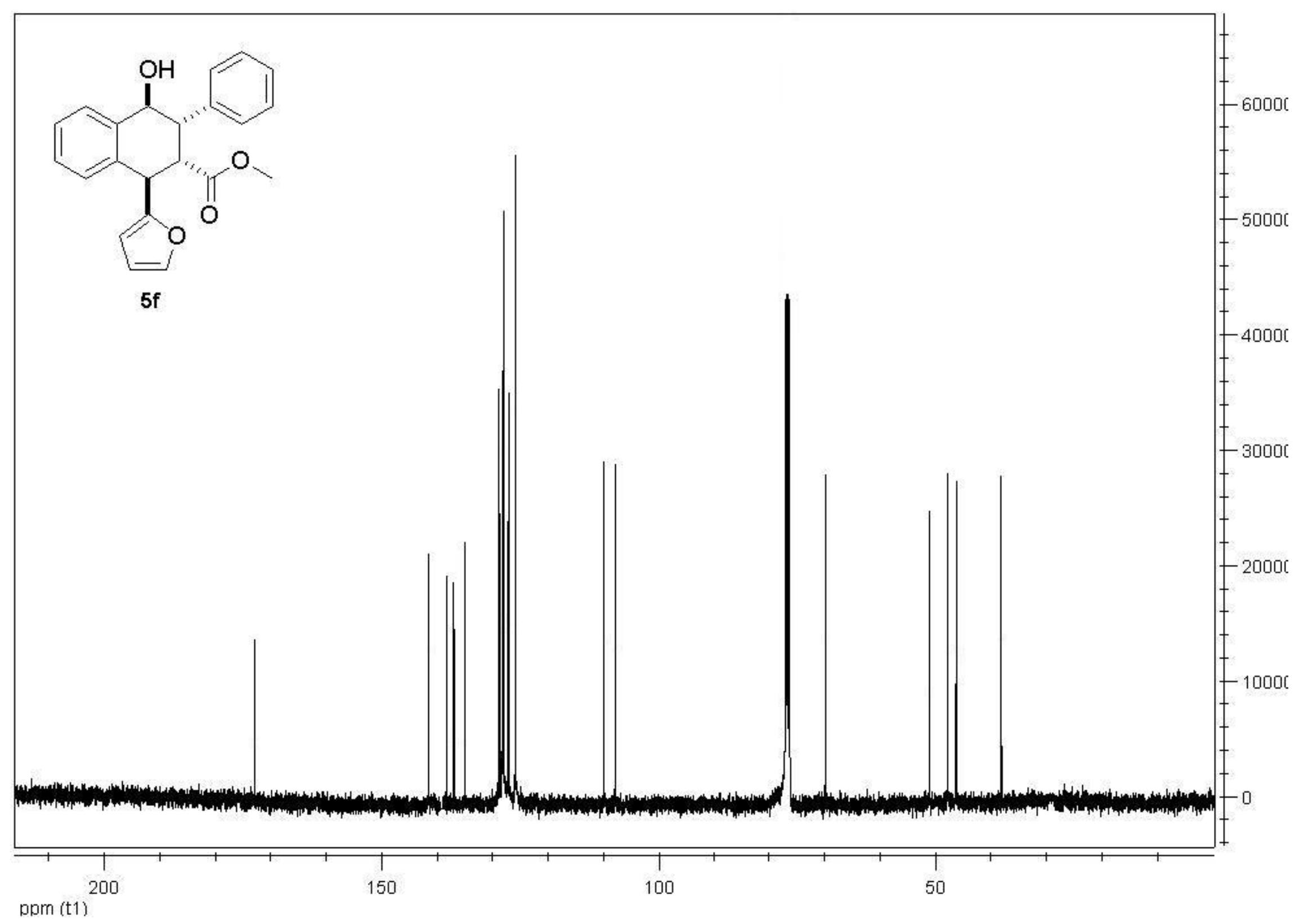




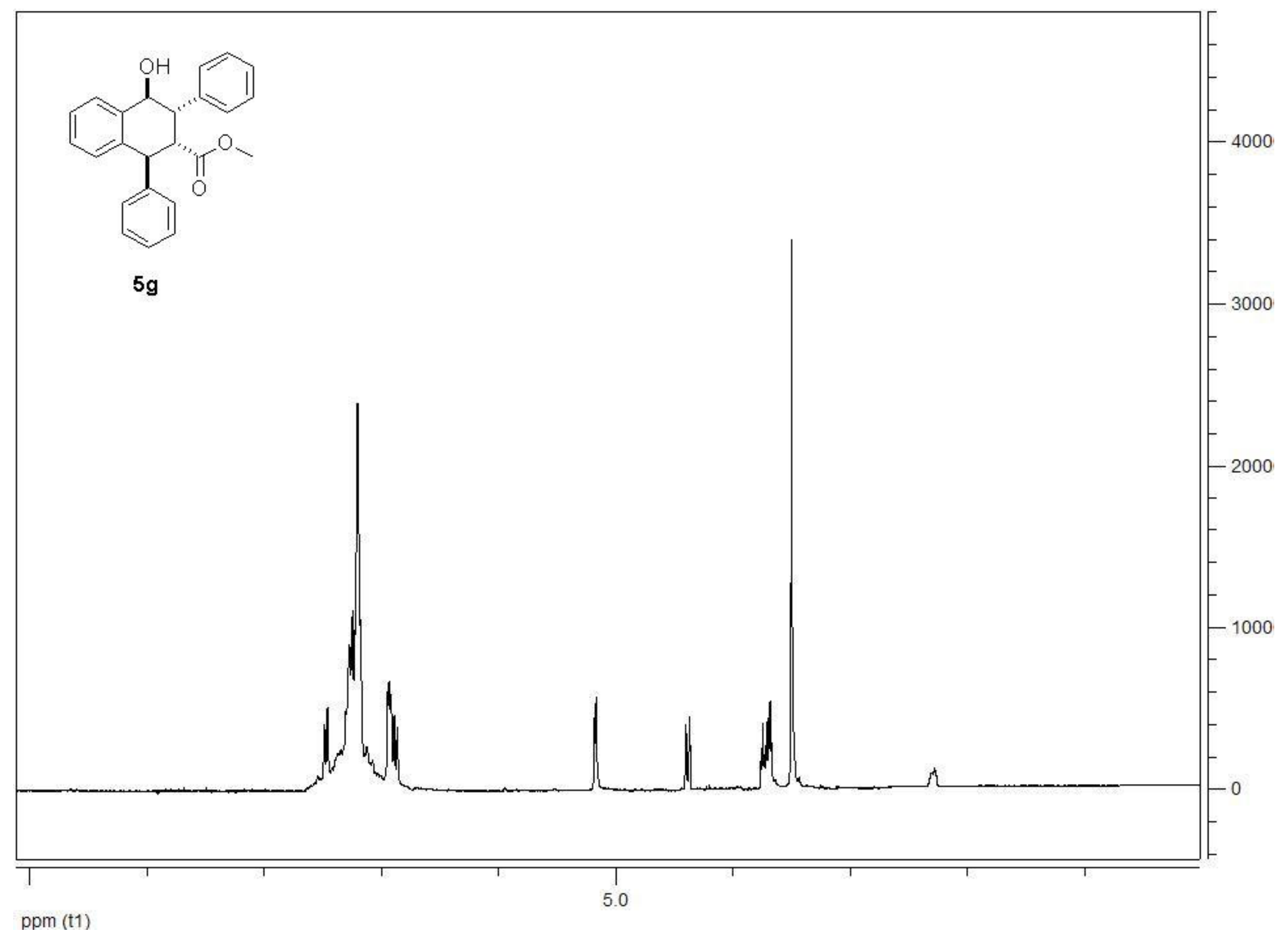




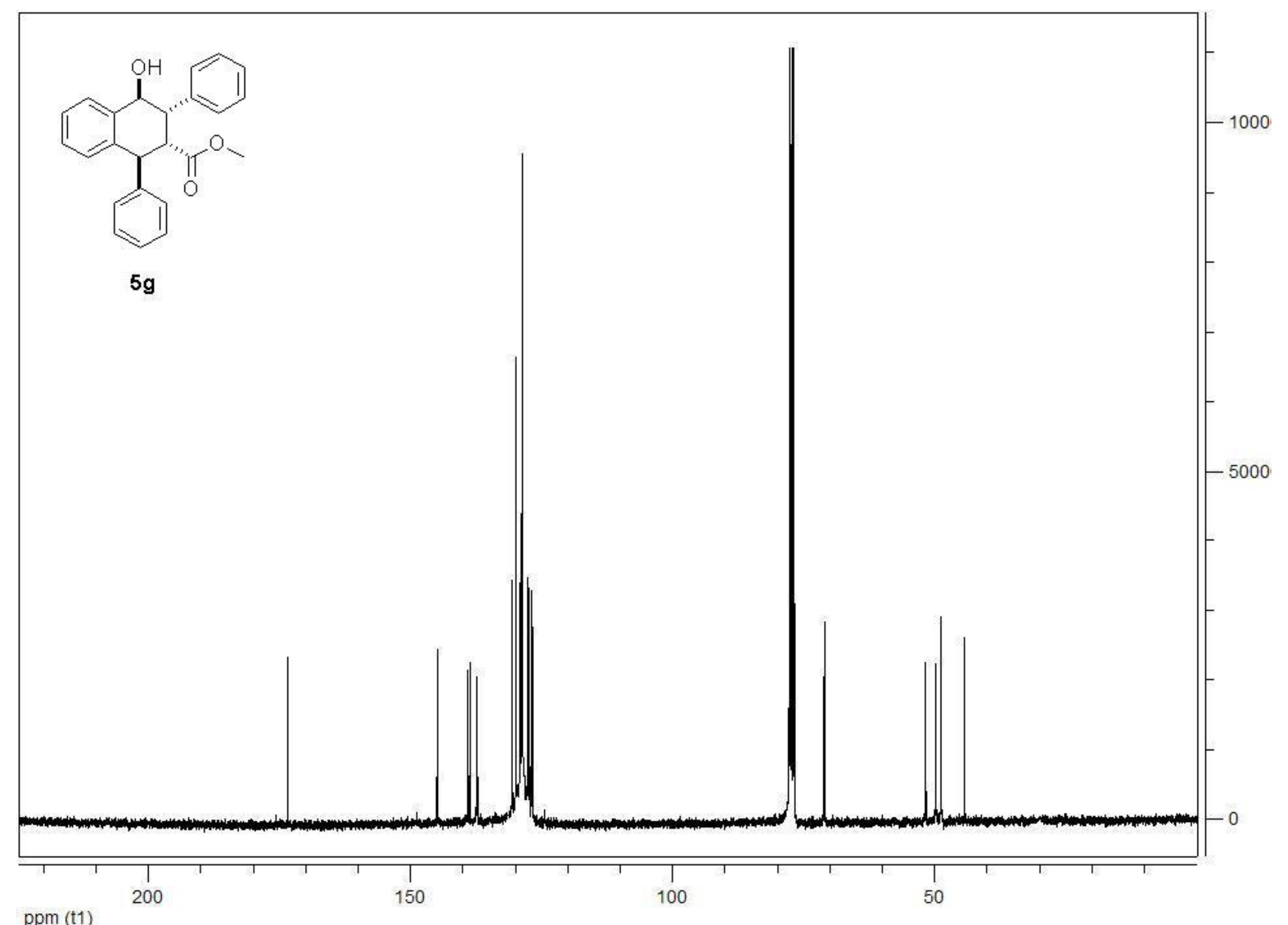




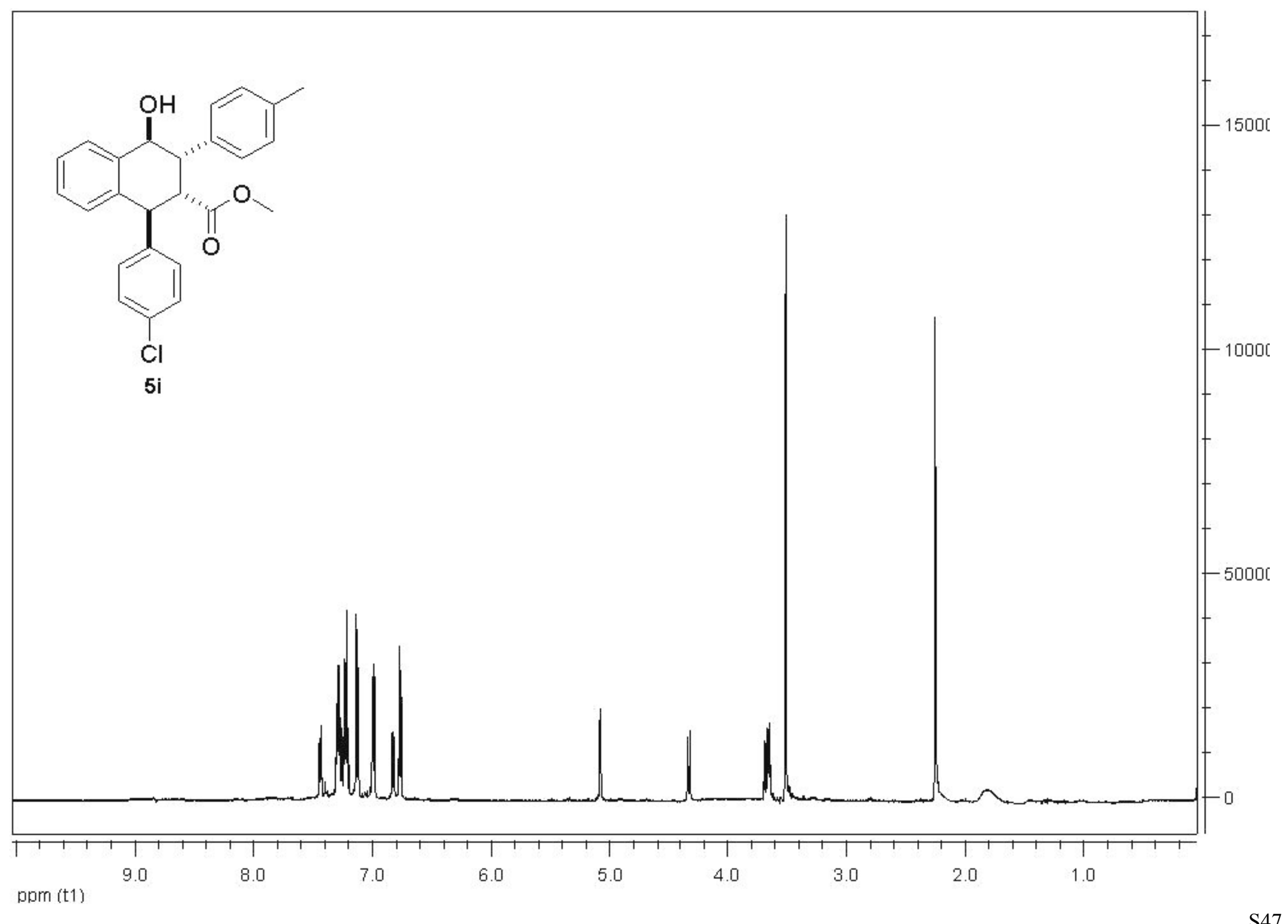




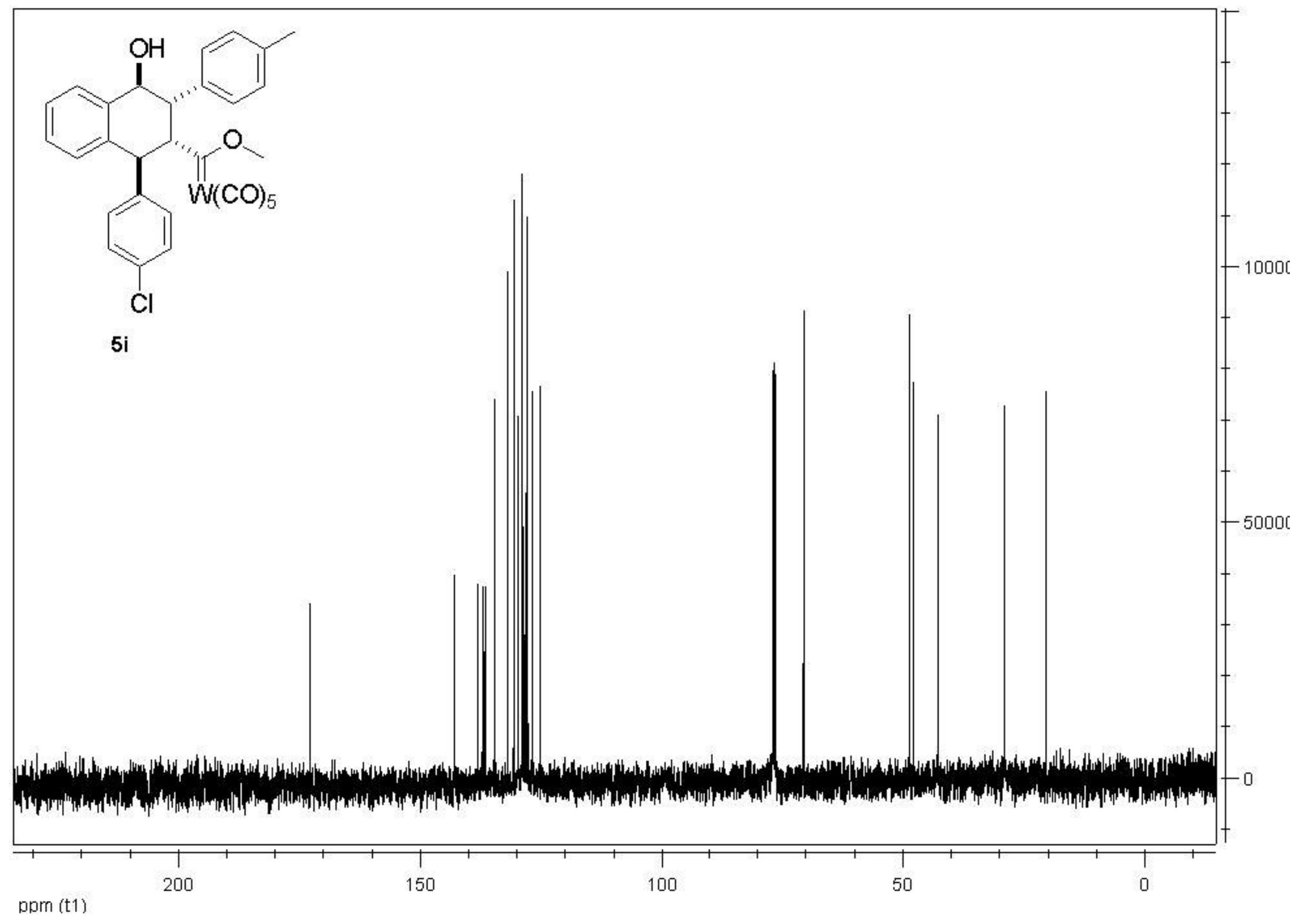




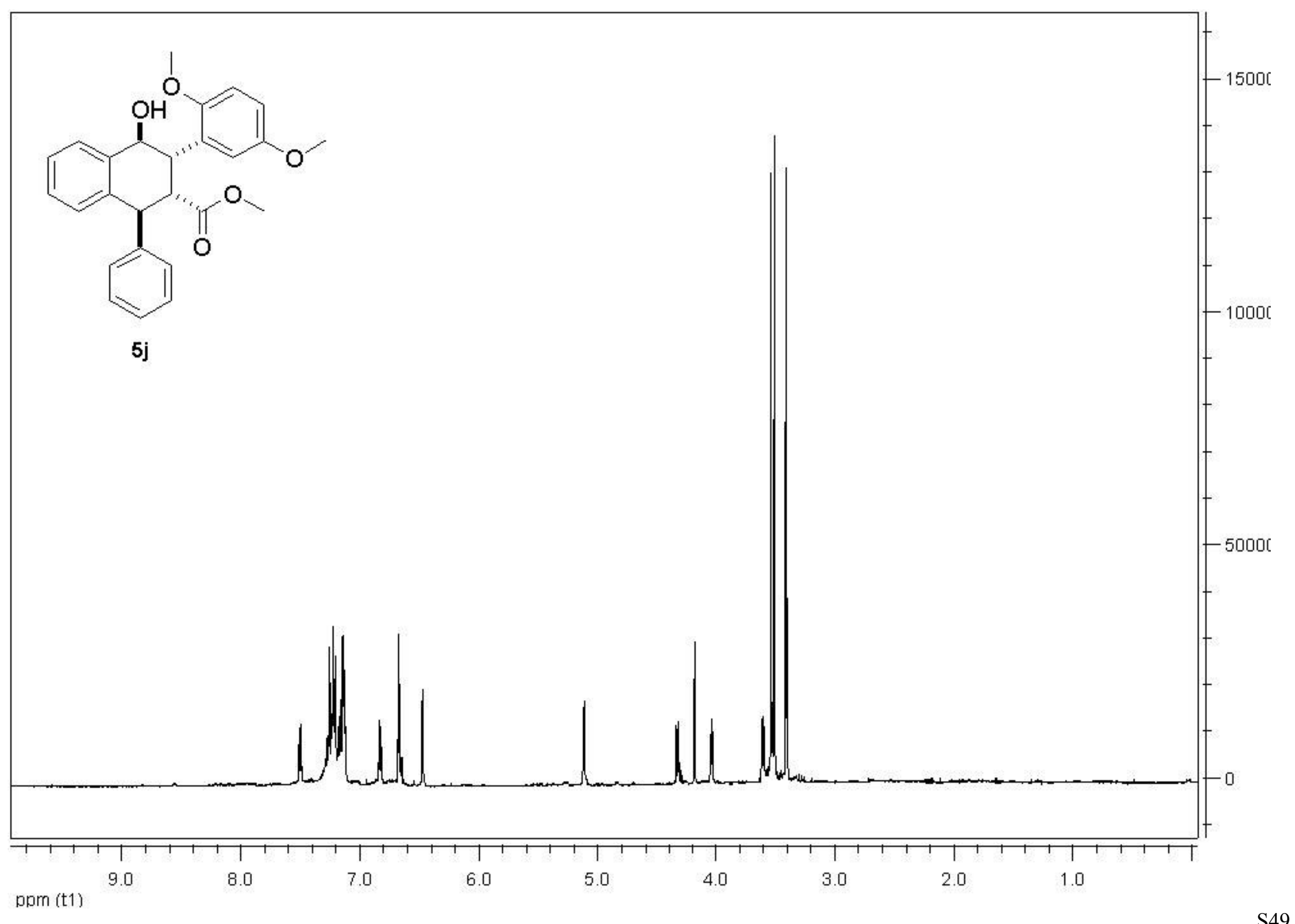




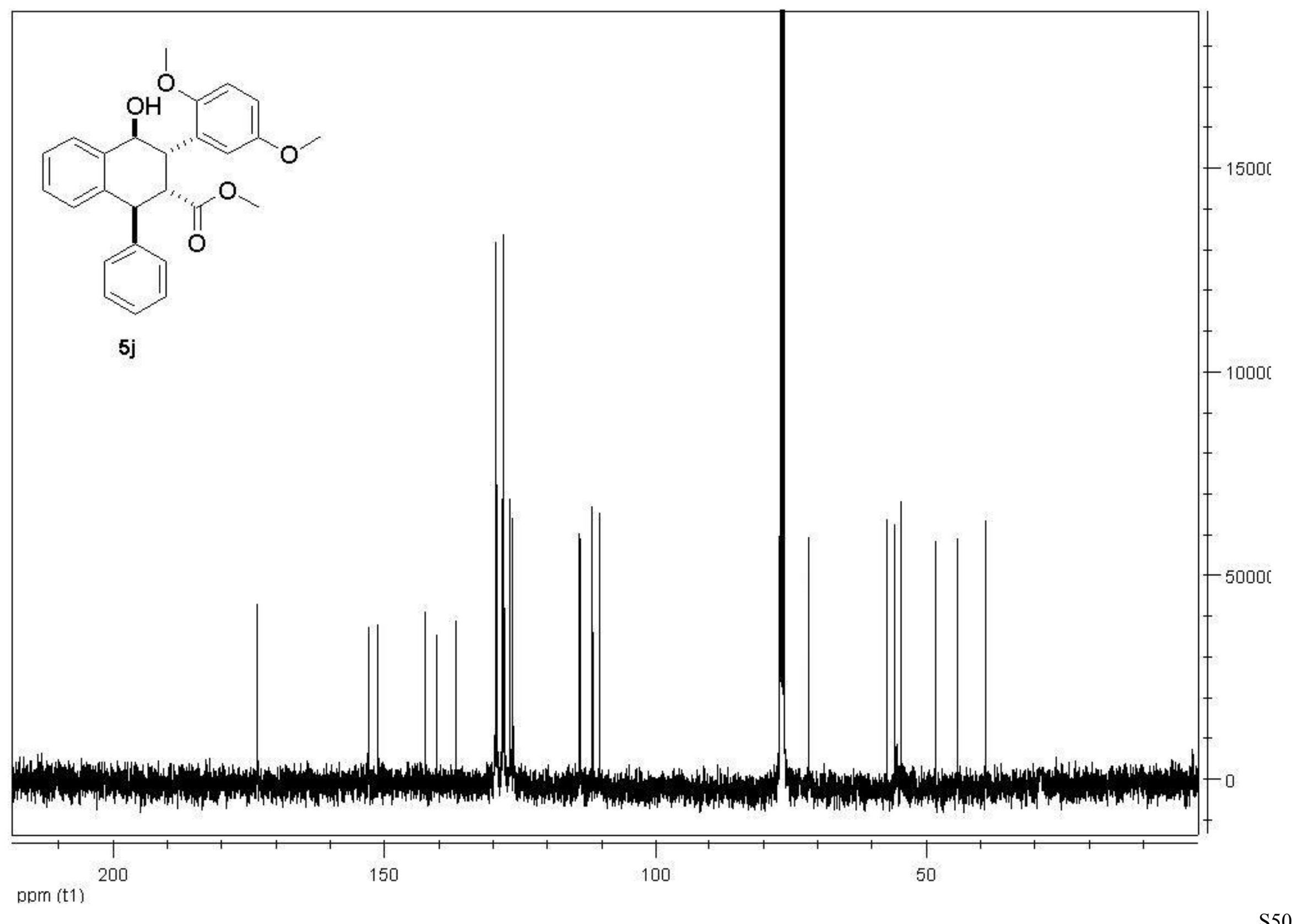




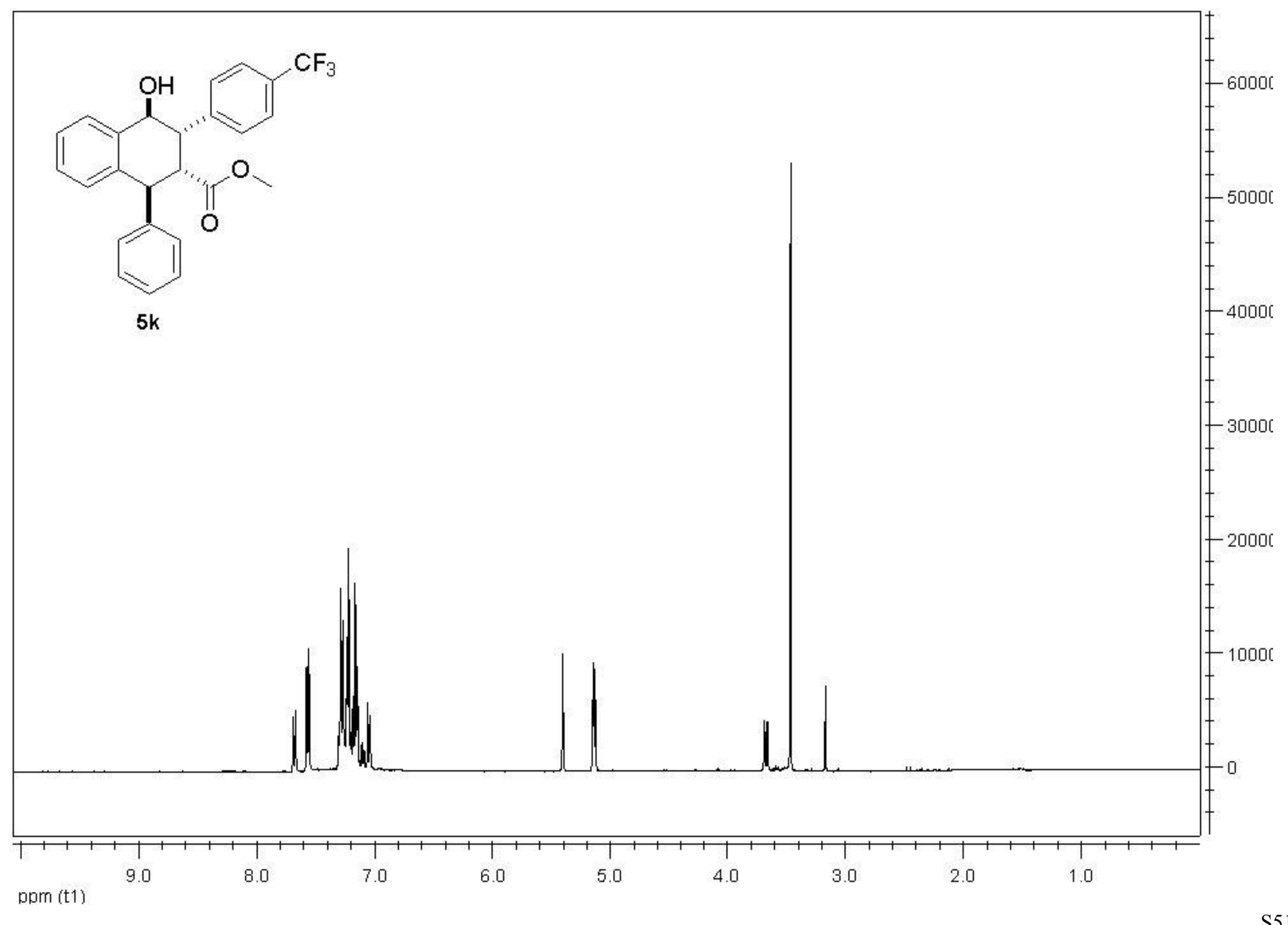




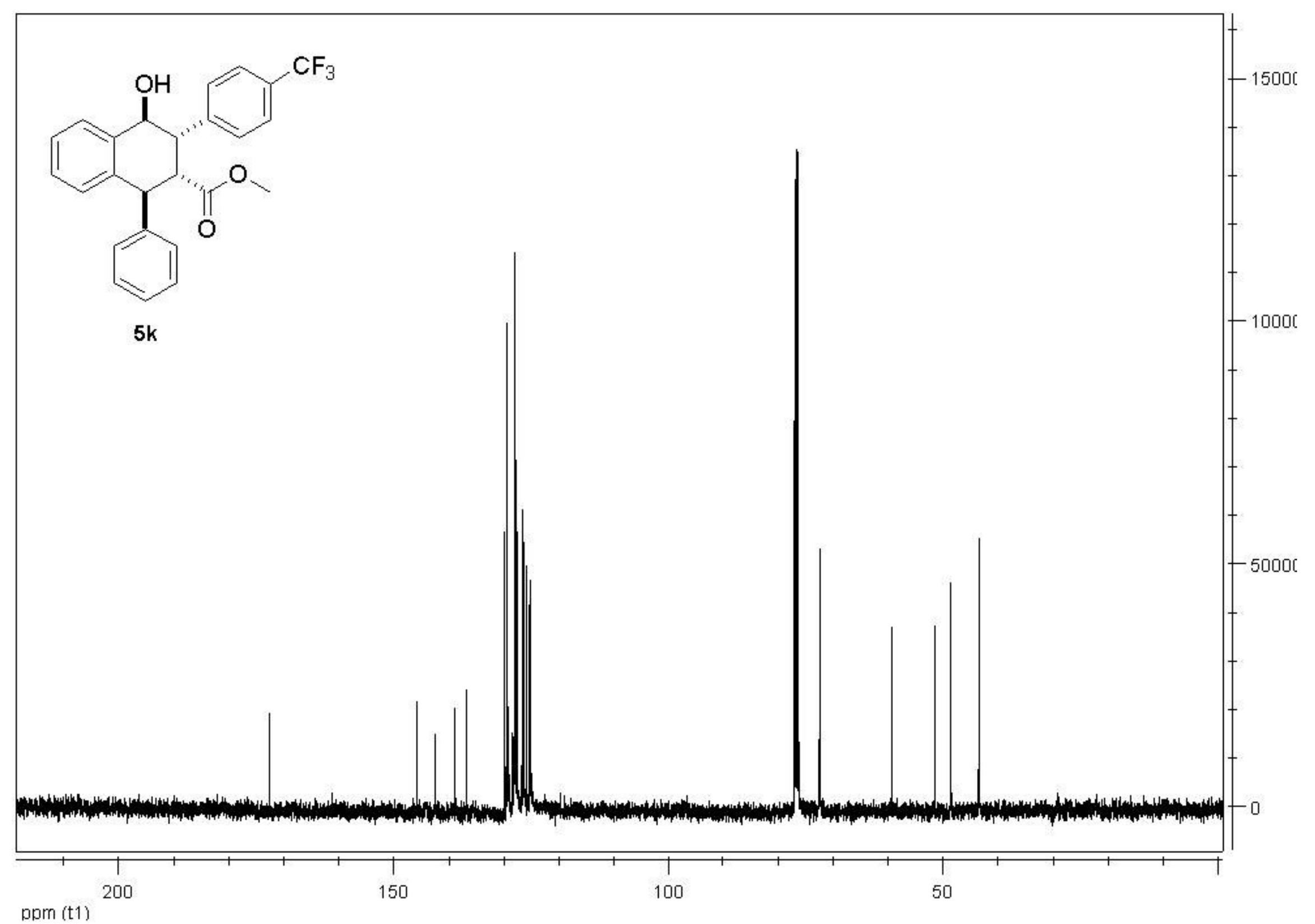

